Research Article

\title{
Mathematical Analysis of Nonlocal Implicit Impulsive Problem under Caputo Fractional Boundary Conditions
}

\author{
Arshad Ali $\mathbb{D}^{1},{ }^{1}$ Vidushi Gupta $\mathbb{D}^{2},{ }^{2}$ Thabet Abdeljawad ${ }^{(D)},{ }^{3,4,5}$ Kamal Shah $\mathbb{D}^{1,3}$ \\ and Fahd Jarad $\mathbb{1 D}^{6}$
}

${ }^{1}$ Department of Mathematics, University of Malakand, Dir (L), Khyber Pakhtunkhwa, Pakistan

${ }^{2}$ Department of Mathematics, Chandigarh University, Chandigarh, Punjab, India

${ }^{3}$ Department of Mathematics and General Sciences, Prince Sultan University, Riyadh, Saudi Arabia

${ }^{4}$ Department of Medical Research, China Medical University, Taichung, Taiwan

${ }^{5}$ Department of Computer Science and Information Engineering, Asia University, Taichung, Taiwan

${ }^{6}$ Department of Mathematics, Cankaya University, Ankara, Turkey

Correspondence should be addressed to Thabet Abdeljawad; tabdeljawad@psu.edu.sa

Received 11 April 2020; Revised 14 August 2020; Accepted 20 August 2020; Published 30 November 2020

Guest Editor: Abdulrahman Al-khedhairi

Copyright (c) 2020 Arshad Ali et al. This is an open access article distributed under the Creative Commons Attribution License, which permits unrestricted use, distribution, and reproduction in any medium, provided the original work is properly cited.

\begin{abstract}
This paper is related to frame a mathematical analysis of impulsive fractional order differential equations (IFODEs) under nonlocal Caputo fractional boundary conditions (NCFBCs). By using fixed point theorems of Schaefer and Banach, we analyze the existence and uniqueness results for the considered problem. Furthermore, we utilize the theory of stability for presenting HyersUlam, generalized Hyers-Ulam, Hyers-Ulam-Rassias, and generalized Hyers-Ulam-Rassias stability results of the proposed scheme. Finally, some applications are offered to demonstrate the concept and results. The whole analysis is carried out by using Caputo fractional derivatives (CFDs).
\end{abstract}

\section{Introduction}

It has been observed that the focus of investigation has shifted from classical integer-order models to fractional-order models. It is because of the fact that many practical systems are excellently described by using fractional-order differential equations (FODEs) instead of classical differential equations. For basic theory and some important applications of fractionalorder derivatives, we refer the readers to see $[1-4]$ and the references therein. Many researchers are devoted to work in this area and made significant contribution in this regard; we refer the readers to the recent work in [5-10].

The study of implicit systems of FODEs with impulsive conditions is quite important as such systems appear in a variety of problems of applied nature, especially in biosciences, economics, engineering, etc. Such problems arise due to abrupt changes in the state of systems like earth quack, fluctuation of pendulum, etc. Here, we refer to some recent papers on impulsive problems [11-16]. The important class of FODEs known as IFODEs has been given much devotion by researchers. One of the most important aspects is investigation of problems under boundary conditions. Such problems mostly occur in engineering. Boundary and initial conditions may be local or nonlocal and both are important, and increasingly many problems have been investigated under these conditions. Replacing the local conditions by nonlocal ones produces a significant effect. This is due to the fact that the measurement computed from a nonlocal condition is usually more precise than the only one measurement given by a local condition. Therefore, the area of nonlocal boundary value problems has also attracted enough attention. In the last two decades, the area of IFODEs has been investigated from various directions including qualitative theory of existence of solution/solutions, stability, and numerical analysis. Therefore, IFODEs have also been investigated under nonlocal boundary conditions. For instance, Gupta and Dabas [17] studied the existence and uniqueness results for a class of IFODEs with nonlocal boundary conditions. 
$\begin{cases}{ }_{0}^{c} \mathscr{D}_{t}^{\varrho} w(t)+f\left(t, w(t), w^{\prime}(t)\right)=0, & \varrho \in(1,2], t \in[0,1], \\ w(0)=0,{ }_{0}^{c} \mathscr{D}_{t}^{\varrho} w(1)=\delta_{0}^{c} \mathscr{D}_{t}^{\varrho} w(\rho), & 0<\xi<1,0<\rho \leq 1 .\end{cases}$
This paper can be considered as generalization of the aforesaid work, in which we discuss existence, uniqueness, and various stability results for the following implicit IFODEs with three point NCFBCs of order $\varrho \in(1,2]$ :

By employing the fixed point technique, the authors obtained the existence and uniqueness results.

$$
\left\{\begin{array}{l}
{ }_{0}^{c} \mathscr{D}_{t}^{\rho} w(t)-f\left(t, w(t),{ }_{0}^{c} \mathscr{D}_{t}^{\rho} w(t)\right)=0, \quad t \in[0, T], \\
\Delta w\left(t_{q}\right)=\mathscr{I}_{q}\left(w\left(t_{q}^{-}\right)\right), \Delta\left({ }_{0}^{c} \mathscr{D}_{t}^{v} w\left(t_{q}\right)\right)=\mathscr{J}_{q}\left(w\left(t_{q}^{-}\right)\right), \quad v \in(0,1], q=1,2, \ldots, \mathbf{r}, \\
w(0)=0,{ }_{0}^{c} \mathscr{D}_{t}^{\varrho} w(T)=\delta_{0}^{c} \mathscr{D}_{t}^{\xi} w(\rho), \quad 0<\delta<\xi<1,0<\rho<1 .
\end{array}\right.
$$

In the proposed problem, the notation ${ }_{0}^{c} \mathscr{D}_{t}^{\varrho},{ }_{0}^{c} \mathscr{D}_{t}^{v}$, and ${ }_{0}^{c} \mathscr{D}_{t}^{\xi}$ represent Caputo fractional derivatives of orders $\varrho, v$, and $\xi$, respectively, where the points 0 and $t$ in the subscript of the differential operator $\mathscr{D}$ are actually the limits of the definite integral involved in the definition of CFD. The function $f:[0, T] \times \mathscr{R} \times \mathscr{R} \longrightarrow \mathscr{R}$ is continuous, where $\mathscr{R}$ is the set of real numbers. The impulsive functions $\mathscr{I}_{q}$ and $\mathscr{I}_{q}$ in $\mathrm{C}(R, R)$ are bounded. For the sequence $0=t^{0<t_{1}<\cdots<t_{\mathrm{r}}<t_{\mathrm{r}+1}=T}$, we have $\Delta w\left(t_{\mathrm{q}}\right)=w\left(t_{\mathrm{q}}^{+}\right)-w\left(t_{\mathrm{q}}^{-}\right)$and $\Delta\left({ }_{0}^{c} \mathscr{D}_{t}^{v} w\left(t_{\mathrm{q}}\right)\right)=\left({ }_{0}^{c} \mathscr{D}_{t}^{v} w\left(t_{\mathrm{q}}^{+}\right)\right)-\left({ }_{0}^{c} \mathscr{D}_{t}^{v} w\left(t_{\mathrm{q}}^{-}\right)\right), w\left(t_{\mathrm{q}}^{+}\right)=\lim _{h \longrightarrow 0}$ $w\left(t_{\mathrm{q}}+h\right)$, and $w\left(t_{\mathrm{q}}^{-}\right)=\lim _{h \longrightarrow 0} w\left(t_{\mathrm{q}}-h\right)$ represents the right-hand and left-hand limits of $w(t)$ at $t=t_{\mathrm{q}}$, respectively, with $w\left(t_{\mathrm{q}}^{-}\right)=w\left(t_{\mathrm{q}}\right)$. The speciality of this proposed problem is that the nonlinear term depends not only on the unknown function but also on its fractional derivative compared with the available results in the literature. This type of study has rarely been discussed in the literature because of the complexity of fractional impulsive surfaces. The further organization of this manuscript is divided into four parts as follows: The second part of the paper demonstrates the preliminary portion in which we recall to readers the basics of used theory, notations, and definitions. The third part presents an existence result by employing Schaefer's fixed point theorem. The fourth section is introduced to analyze and study several stability results of the considered problem, and the last section is provided to illustrate the applications of the obtained results.

\section{Preliminaries}

We take $\mathcal{S}=[0, T], \mathcal{S}_{0}=\left[0, t_{1}\right]$, and $\mathcal{S}_{\mathrm{q}}=\left(t_{q}, t_{q+1}\right]$. We introduce the following space of piecewise continuous functions by

$$
\begin{aligned}
\mathscr{B} & =P C(\mathcal{S}, \mathscr{R})=\{w: \mathcal{S} \longrightarrow \mathscr{R} \mid w \in C(\mathcal{S}), q \\
& \left.=1,2, \ldots, \mathbf{r}, w\left(t_{\mathrm{q}}^{+}\right), w\left(t_{\mathrm{q}}^{-}\right) \text {exist for } q=1,2, \ldots, \mathbf{r}\right\}
\end{aligned}
$$

where $\mathscr{B}$ is the Banach space corresponding to the norm $\|w\|_{\mathscr{B}}=\max _{t \in \mathcal{S}}|w(t)|$.
Definition 1 (see [18]). The fractional order integral of function $g \in L^{1}\left([a, b], \mathscr{R}^{+}\right)$of order $\varrho \in \mathscr{R}^{+}$is defined by

$$
I_{a}^{\varrho} g(t)=\int_{a}^{t} \frac{(t-\eta)^{\varrho-1}}{\Gamma(\varrho)} g(\eta) \mathrm{d} \eta
$$

where $\Gamma$ is the gamma function.

Definition 2 (see [18]). For a function $g$ given on interval $[a, b]$, the CFD of $g$ is defined by

$$
{ }_{0}^{c} \mathscr{D}_{t}^{\varrho} g(t)=\frac{1}{\Gamma(n-\varrho)} \int_{a}^{t}(t-\eta)^{n-\varrho-1} g^{(n)}(\eta) \mathrm{d} \eta,
$$

where $n=[\varrho]+1$.

Let there exist constants $\beta>0$ and $\epsilon>0$ and a nondecreasing function $\Phi \in C(\mathcal{S}, \mathscr{R})$, such that the following inequalities exist for $q=1,2, \ldots, \mathbf{r}$ :

$$
\begin{aligned}
& \left\{\begin{array}{l}
\left|{ }_{0}^{c} \mathscr{D}_{t}^{\varrho} h(t)-f\left(t, h(t),{ }_{0}^{c} \mathscr{D}_{t}^{\varrho} h(t)\right)\right| \leq \epsilon, \quad t \in \mathcal{S}, \\
\left|\Delta h\left(t_{q}\right)-\mathscr{I}_{q}\left(h\left(t_{q}^{-}\right)\right)\right| \leq \epsilon, \\
\left|\Delta\left({ }_{0}^{c} \mathscr{D}_{t}^{\varrho} h\left(t_{q}\right)\right)-\mathscr{J}_{q}\left(h\left(t_{q}^{-}\right)\right)\right| \leq \epsilon,
\end{array}\right. \\
& \left\{\begin{array}{l}
\left|{ }_{0}^{c} \mathscr{D}_{t}^{\varrho} h(t)-f\left(t, h(t),{ }_{0}^{c} \mathscr{D}_{t}^{\varrho} h(t)\right)\right| \leq \Phi(t), \quad t \in \mathcal{S}, \\
\left|\Delta h\left(t_{q}\right)-\mathscr{I}_{q}\left(h\left(t_{q}^{-}\right)\right)\right| \leq \beta, \\
\mid \Delta\left({ }_{0}^{c} \mathscr{D}_{t}^{\varrho}\left(h\left(t_{q}\right)\right)-\mathscr{J}_{q}\left(h\left(t_{q}^{-}\right)\right)\right) \leq \beta,
\end{array}\right. \\
& \left\{\begin{array}{l}
\left|{ }_{0}^{c} \mathscr{D}_{t}^{\varrho} h(t)-f\left(t, h(t),{ }_{0}^{c} \mathscr{D}_{t}^{\varrho} h(t)\right)\right| \leq \epsilon \Phi(t), \quad t \in \mathcal{S}, \\
\left|\Delta h\left(t_{q}\right)-\mathscr{I}_{q}\left(h\left(t_{q}^{-}\right)\right)\right| \leq \epsilon \beta, \\
\left|\Delta\left({ }_{0}^{c} \mathscr{D}_{t}^{v} h\left(t_{q}\right)\right)-\mathscr{J}_{q}\left(h\left(t_{q}^{-}\right)\right)\right| \leq \epsilon \beta .
\end{array}\right.
\end{aligned}
$$

Definition 3 (see [19]). If for $\epsilon>0$ there exists a constant $\mathscr{C}>0$ such that for any solution $h \in \mathscr{B}$ of inequality (6), there is a unique solution $w \in \mathscr{B}$ of problem (2) which satisfies

$$
|h(t)-w(t)| \leq \mathscr{C} \epsilon, \quad t \in \mathcal{S},
$$

then problem (2) is called Hyers-Ulam stable. 
Definition 4 (see [19]). If for $\epsilon>0$ and set of positive real numbers $\mathscr{R}^{+}$there exists $\psi \in C\left(\mathscr{R}^{+}, \mathscr{R}^{+}\right)$, such that for any solution $h \in \mathscr{B}$ of inequality (6), there is a unique solution $w \in \mathscr{B}$ of problem (2) which satisfies

$$
|h(t)-w(t)| \leq \psi(\varepsilon), \quad t \in \mathcal{S},
$$

then problem (2) is called generalized Hyers-Ulam stable.

Definition 5 (see [19]). If for $\epsilon>0$ there exists a real number $\mathscr{C}>0$, such that for any solution $h \in \mathscr{B}$ of inequality (8), there is a unique solution $w \in \mathscr{B}$ of problem (2) which satisfies

$$
|h(t)-w(t)| \leq \mathscr{C} \mathcal{E}(\Phi(t)+\psi), \quad t \in \mathcal{S},
$$

then problem (2) is called Hyers-Ulam-Rassias stable with respect to $(\Phi, \psi)$.

Definition 6 (see [19]). If there exists constant $\mathscr{C}>0$, such that for any solution $h \in \mathscr{B}$ of inequality (7), there is a unique solution $w \in \mathscr{B}$ of problem (2) which satisfies

$$
|h(t)-w(t)| \leq \mathscr{C}(\Phi(t)+\psi), \quad t \in \mathcal{S}
$$

then problem (2) is called generalized Hyers-Ulam-Rassias stable with respect to $(\Phi, \psi)$.

Here, it is to be noted that Definitions 3-6 have been adopted from the paper [19].

Remark 1. The function $h \in \mathscr{B}$ is called a solution for inequality (6) if there exists a function $\phi \in \mathscr{B}$ together with a sequence $\phi_{q}$, where $q=1,2, \ldots, \mathbf{r}$ (which depends on $h$ ) such that (i) $|\phi(t)| \leq \epsilon,\left|\phi_{\mathrm{q}}\right| \leq \epsilon, t \in \mathcal{S}, q=1,2, \ldots, \mathbf{r}$

(ii) ${ }_{0}^{c} \mathscr{D}_{t}^{\varrho} h(t)=f\left(t, h(t),{ }_{0}^{c} \mathscr{D}_{t}^{\varrho} h(t)\right)+\phi(t), t \in \mathcal{S}, q=$ $1,2, \ldots, \mathbf{r}$

(iii) $\Delta h\left(t_{q}\right)=\mathscr{I}_{q}\left(h\left(t_{q}^{-}\right)\right)+\phi_{q} t \in \mathcal{S}, q=1,2, \ldots, \mathbf{r}$

(iv) $\Delta\left({ }_{0}^{c} \mathscr{D}_{t}^{\nu} h\left(t_{q}\right)\right)=\mathscr{J}_{q}\left(h\left(t_{q}^{-}\right)\right)+\phi_{q}, t \in \mathcal{S}, q=1,2, \ldots, \mathbf{r}$

Remark 2. A function $h \in \mathscr{B}$ is a solution of inequality (8) if there exists a function $\phi \in \mathscr{B}$ and a sequence $\phi_{q}$, where $q=$ $1,2, \ldots, \mathbf{r}$ (which depends on $h$ ) such that

(i) $|\phi(t)| \leq \epsilon \theta(t),\left|\phi_{q}\right| \leq \epsilon \varphi, t \in \mathcal{S}, q=1,2, \ldots, \mathbf{r}$

(ii) ${ }_{0}^{c} \mathscr{D}_{t}^{\varrho} h(t)=f\left(t, h(t),{ }_{0}^{c} \mathscr{D}_{t}^{\varrho} h(t)\right)+\phi(t), t \in \mathcal{S}, q=$ $1,2, \ldots, \mathbf{r}$

(iii) $\Delta h\left(t_{\mathrm{q}}\right)=\mathscr{I}_{\mathrm{q}}\left(h\left(t_{\mathrm{q}}^{-}\right)\right)+\phi_{\mathrm{q}}, t \in \mathcal{S}, q=1,2, \ldots, \mathbf{r}$

(iv) $\Delta\left({ }_{0}^{c} \mathscr{D}_{t}^{v} h\left(t_{q}\right)\right)=\mathscr{J}_{q}\left(h\left(t_{q}^{-}\right)\right)+\phi_{q}, t \in \mathcal{S}, q=1,2, \ldots, \mathbf{r}$

Lemma 1 (see [20]). For $\mathrm{\varrho}>0$, the given result holds:

$$
{ }_{0} I_{t}^{\varrho}\left({ }_{0}^{c} \mathscr{D}_{t}^{\varrho} g(t)\right)=g(t)-\sum_{i=0}^{n-1} c_{i} t^{i}, \quad \text { where } n=[\varrho]+1 .
$$

To investigate the nonlinear IFODE2, we first consider the associated linear problem and obtain its solution.

Lemma 2. Let $\varrho \in(1,2)$ and $\sigma:[0, T] \longrightarrow \mathscr{R}$ be continuous. A function $w(t)$ is a solution of the fractional integral equation:

$$
w(t)=\left\{\begin{array}{l}
\int_{0}^{t} \frac{(t-\eta)^{\varrho-1}}{\Gamma(\varrho)} \sigma(\eta) \mathrm{d} \eta-t\left[\frac { \Gamma ( 2 - \xi ) } { ( \delta \rho ^ { 1 - \xi } - T ^ { 1 - \xi } ) } \left(\delta \int_{0}^{\rho} \frac{(\rho-\eta)^{\varrho-\xi-1}}{\Gamma(\varrho-\xi)} \sigma(\eta) \mathrm{d} \eta\right.\right. \\
\left.\left.-\int_{0}^{T} \frac{(T-\eta)^{\varrho-\xi-1}}{\Gamma(\varrho-\xi)} \sigma(\eta) \mathrm{d} \eta\right)+\sum_{i=1}^{\mathrm{q}}\left(\frac{\Gamma(2-\nu)}{t_{i}^{1-v}} \mathscr{J}_{i}\left(w\left(t_{i}^{-}\right)\right)\right)\right], \quad t \in\left[0, t_{1}\right], q=1,2, \ldots, \mathbf{r}, \\
\int_{0}^{t} \frac{(t-\eta)^{\varrho-1}}{\Gamma(\varrho)} \sigma(\eta) \mathrm{d} \eta+\sum_{i=1}^{\mathrm{q}} \mathscr{J}_{i}\left(w\left(t_{i}^{-}\right)\right)-t\left[\frac { \Gamma ( 2 - \xi ) } { ( \delta \rho ^ { 1 - \xi } - T ^ { 1 - \xi } ) } \left(\delta \int_{0}^{\rho} \frac{(\rho-\eta)^{\varrho-\xi-1}}{\Gamma(\varrho-\xi)} \sigma(\eta) \mathrm{d} \eta\right.\right. \\
\left.\left.-\int_{0}^{T} \frac{(T-\eta)^{\varrho-\xi-1}}{\Gamma(\varrho-\xi)} \sigma(\eta) \mathrm{d} \eta\right)+\sum_{i=1}^{\mathrm{q}}\left(\frac{\Gamma(2-\nu)}{t_{i}^{1-v}} \mathscr{J}_{i}\left(w\left(t_{i}^{-}\right)\right)\right)\right]+\sum_{i=1}^{\mathrm{q}}\left(t-t_{i}\right) \\
\cdot\left(\frac{\Gamma(2-\nu)}{\left.t_{i}^{1-v} \mathscr{J}_{i}\left(w\left(t_{i}^{-}\right)\right)\right),} t \in\left(t_{q}, t_{q+1}\right], q=1,2, \ldots, \mathbf{r},\right.
\end{array}\right.
$$

if and only if $w(t)$ is a solution of the following BVP: 


$$
\left\{\begin{array}{l}
{ }_{0}^{c} \mathscr{D}_{t}^{\varrho} w(t)-\sigma(t)=0, \quad \varrho \in(1,2], \\
\Delta w\left(t_{q}\right)=\mathscr{I}_{q}\left(w\left(t_{q}^{-}\right)\right), \quad \Delta\left({ }_{0}^{c} \mathscr{D}_{t}^{v} w\left(t_{q}\right)\right)=\mathscr{J}_{q}\left(w\left(t_{q}^{-}\right)\right), \quad v \in(0,1], q=1,2, \ldots, \mathbf{r}, \\
w(0)=0,{ }_{0}^{c} \mathscr{D}_{t}^{\xi} w(T)=\delta_{0}^{c} \mathscr{D}_{t}^{\xi} w(\rho), \quad 0<\delta<\xi<1 .
\end{array}\right.
$$

Proof. Let for $t \in\left[0, t_{1}\right), w(t)$ be the solution of (15), then by Lemma 1, we have

$$
w(t)=\int_{0}^{t} \frac{(t-\eta)^{\varrho^{-1}}}{\Gamma(\varrho)} \sigma(\eta) \mathrm{d} \eta-c_{0}-c_{1} t .
$$

Using the condition $w(0)=0$, we get

$$
c_{0}=0 \text {. }
$$

Substituting $c_{0}$ in (16), we get

$$
w(t)=\int_{0}^{t} \frac{(t-\eta)^{\varrho-1}}{\Gamma(\varrho)} \sigma(\eta) \mathrm{d} \eta-c_{1} t
$$

For $t \in\left(t_{1}, t_{2}\right]$, we get

$$
w(t)=\int_{0}^{t} \frac{(t-\eta)^{\varrho^{-1}}}{\Gamma(\varrho)} \sigma(\eta) \mathrm{d} \eta-c_{2}-c_{3} t .
$$

Applying the impulsive condition $\Delta w\left(t_{1}\right)=\mathscr{I}_{1}\left(w\left(t_{1}^{-}\right)\right)$, we get

$$
\begin{aligned}
\mathscr{I}_{1}\left(w\left(t_{1}^{-}\right)\right) & =-c_{2}-c_{3} t_{1}+c_{1} t_{1}, \\
-c_{2} & =\mathscr{I}_{1}\left(w\left(t_{1}^{-}\right)\right)+c_{3} t_{1}-c_{1} t_{1} .
\end{aligned}
$$

Substituting $c_{2}$ in (19), we get

$$
w(t)=\int_{0}^{t} \frac{(t-\eta)^{\varrho^{-1}}}{\Gamma(\varrho)} \sigma(\eta) \mathrm{d} \eta+\mathscr{I}_{1}\left(w\left(t_{1}^{-}\right)\right)+c_{3} t_{1}-c_{1} t_{1}-c_{3} t,
$$

$$
w(t)=\int_{0}^{t} \frac{(t-\eta)^{\varrho-1}}{\Gamma(\varrho)} \sigma(\eta) \mathrm{d} \eta+\mathscr{I}_{1}\left(w\left(t_{1}^{-}\right)\right)-c_{1} t_{1}+c_{3}\left(t_{1}-t\right)
$$

From equations (18) and (22), we get

$$
\begin{aligned}
& { }_{0}^{c} \mathscr{D}_{t}^{\nu} w(t)=\frac{1}{\Gamma(\varrho-\nu)} \int_{0}^{t}(t-\eta)^{\varrho-\nu-1} \sigma(\eta) \mathrm{d} \eta-c_{3} \frac{t^{1-\nu}}{\Gamma(2-\nu)} \\
& { }_{0}^{c} \mathscr{D}_{t}^{v} w(t)=\frac{1}{\Gamma(\varrho-\nu)} \int_{0}^{t}(t-\eta)^{\varrho-\nu-1} \sigma(\eta) \mathrm{d} \eta-c_{1} \frac{t^{1-\nu}}{\Gamma(2-\nu)} .
\end{aligned}
$$

Now, using the impulsive condition $\Delta\left({ }_{0}^{c} \mathscr{D}_{t}^{v} w\left(t_{1}\right)\right)=$ $\mathscr{J}_{1}\left(w\left(t_{1}^{-}\right)\right)$, we get

$$
\begin{aligned}
\mathscr{J}_{1}\left(w\left(t_{1}^{-}\right)\right) & =-c_{3} \frac{t_{1}^{1-v}}{\Gamma(2-v)}+c_{1} \frac{t_{1}^{1-v}}{\Gamma(2-v)}, \\
c_{3} & =-\frac{\Gamma(2-v)}{t_{1}^{1-v}} \mathscr{J}_{1}\left(w\left(t_{1}^{-}\right)\right)+c_{1} .
\end{aligned}
$$

Substituting $c_{3}$ in (22), we get

$$
\begin{aligned}
w(t)= & \int_{0}^{t} \frac{(t-\eta)^{\varrho-1}}{\Gamma(\varrho)} \sigma(\eta) \mathrm{d} \eta+\mathscr{I}_{1}\left(w\left(t_{1}^{-}\right)\right) \\
& +\left(t-t_{1}\right) \frac{\Gamma(2-\nu)}{t_{1}^{1-v}} \mathscr{J}_{1}\left(w\left(t_{1}^{-}\right)\right)-c_{1} t .
\end{aligned}
$$

For $t \in\left(t_{2}, t_{3}\right]$, we get

$$
w(t)=\int_{0}^{t} \frac{(t-\eta)^{\varrho-1}}{\Gamma(\varrho)} \sigma(\eta) \mathrm{d} \eta-c_{4}-c_{5} t .
$$

Applying the impulsive condition $\Delta w\left(t_{2}\right)=\mathscr{I}_{2}\left(w\left(t_{2}^{-}\right)\right)$ in (26) and (25), we get

$$
\begin{aligned}
\mathscr{I}_{2}\left(w\left(t_{2}^{-}\right)\right)= & -c_{4}-c_{5} t_{2}-\mathscr{I}_{1}\left(w\left(t_{1}^{-}\right)\right) \\
& -\left(t_{2}-t_{1}\right) \frac{\Gamma(2-v)}{t_{1}^{1-v}} \mathscr{J}_{1}\left(w\left(t_{1}^{-}\right)\right)+c_{1} t_{2}, \\
-c_{4}= & \mathscr{I}_{2}\left(w\left(t_{2}^{-}\right)\right)+c_{5} t_{2}+\mathscr{I}_{1}\left(w\left(t_{1}^{-}\right)\right) \\
& +\left(t_{2}-t_{1}\right) \frac{\Gamma(2-\nu)}{t_{1}^{1-v}} \mathscr{J}_{1}\left(w\left(t_{1}^{-}\right)\right)-c_{1} t_{2} .
\end{aligned}
$$

Substituting $c_{4}$ in (26), we get

$$
\begin{aligned}
w(t)= & \int_{0}^{t} \frac{(t-\eta)^{\varrho-1}}{\Gamma(\varrho)} \sigma(\eta) \mathrm{d} \eta+\mathscr{I}_{2}\left(w\left(t_{2}^{-}\right)\right)+c_{5} t_{2}+\mathscr{I}_{1} \\
& \cdot\left(w\left(t_{1}^{-}\right)\right)+\left(t_{2}-t_{1}\right) \frac{\Gamma(2-\nu)}{t_{1}^{1-v}} \mathscr{J}_{1}\left(w\left(t_{1}^{-}\right)\right)-c_{1} t_{2}-c_{5} t .
\end{aligned}
$$

By equations (25) and (28), we get

$$
\begin{aligned}
{ }_{0}^{c} \mathscr{D}_{t}^{\nu} w(t)= & \frac{1}{\Gamma(\varrho-\nu)} \int_{0}^{t}(t-\eta)^{\varrho-\nu-1} \sigma(\eta) \mathrm{d} \eta-c_{5} \frac{t^{1-\nu}}{\Gamma(2-\nu)} \\
{ }_{0}^{c} \mathscr{D}_{t}^{\nu} w(t)= & \frac{1}{\Gamma(\varrho-\nu)} \int_{0}^{t}(t-\eta)^{\varrho-\nu-1} \sigma(\eta) \mathrm{d} \eta \\
& +\left(\frac{\Gamma(2-\nu)}{t_{1}^{1-v}} \mathscr{J}_{1}\left(w\left(t_{1}^{-}\right)\right)-c_{1}\right) \frac{t^{1-\nu}}{\Gamma(2-\nu)}
\end{aligned}
$$

Now, using the impulsive condition $\Delta\left({ }_{0}^{c} \mathscr{D}_{t}^{v} w\left(t_{2}\right)\right)=$ $\mathscr{J}_{2}\left(w\left(t_{2}^{-}\right)\right)$, we get 


$$
\begin{gathered}
\mathscr{J}_{2}\left(w\left(t_{2}^{-}\right)\right)=-c_{5} \frac{t_{2}^{1-v}}{\Gamma(2-v)}-\left(\frac{\Gamma(2-v)}{t_{1}^{1-v}} \mathscr{J}_{1}\left(w\left(t_{1}^{-}\right)\right)-c_{1}\right) \frac{t_{2}^{1-v}}{\Gamma(2-v)}, \\
c_{5}=-\frac{\Gamma(2-v)}{t_{1}^{1-v}} \mathscr{J}_{1}\left(w\left(t_{1}^{-}\right)\right)-\frac{\Gamma(2-v)}{t_{2}^{1-v}} \mathscr{J}_{2}\left(w\left(t_{2}^{-}\right)\right)+c_{1} .
\end{gathered}
$$

Substituting $c_{5}$ in (28), we get

$$
\begin{aligned}
w(t)= & \int_{0}^{t} \frac{(t-\eta)^{\varrho-1}}{\Gamma(\varrho)} \sigma(\eta) \mathrm{d} \eta+\mathscr{I}_{1}\left(w\left(t_{1}^{-}\right)\right)+\mathscr{I}_{2}\left(w\left(t_{2}^{-}\right)\right) \\
& +\frac{\Gamma(2-\nu)}{t_{1}^{1-v}} \mathscr{J}_{1}\left(w\left(t_{1}^{-}\right)\right)\left(t-t_{1}\right) \\
& +\frac{\Gamma(2-\nu)}{t_{2}^{1-v}} \mathscr{J}_{2}\left(w\left(t_{2}^{-}\right)\right)\left(t-t_{2}\right)-c_{1} t .
\end{aligned}
$$

Similarly for $t \in \mathcal{S}_{\mathrm{q}}$, we get

$$
\begin{aligned}
w(t)= & \int_{0}^{t} \frac{(t-\eta)^{\varrho-1}}{\Gamma(\varrho)} \sigma(\eta) \mathrm{d} \eta+\sum_{1=1}^{\mathrm{q}} \mathscr{I}_{1}\left(w\left(t_{1}^{-}\right)\right)-c_{1} t \\
& +\sum_{1=1}^{\mathrm{q}}\left(t-t_{1}\right)\left(\frac{\Gamma(2-v)}{t_{i}^{1-v}} \mathscr{J}_{1}\left(w\left(t_{1}^{-}\right)\right)\right),
\end{aligned}
$$

$$
\begin{aligned}
{ }_{0}^{c} \mathscr{D}_{t}^{v} w(t)= & \int_{0}^{t} \frac{(t-\eta)^{\varrho^{-\xi}-1}}{\Gamma(\varrho-\xi)} \sigma(\eta) \mathrm{d} \eta-c_{1} \frac{t^{1-\xi}}{\Gamma(2-\xi)} \\
& +\left(\frac{t^{1-\xi}}{\Gamma(2-\xi)}\right) \sum_{1=1}^{\mathrm{q}}\left(\frac{\Gamma(2-\nu)}{t_{i}^{1-v}} \mathscr{J}_{1}\left(w\left(t_{1}^{-}\right)\right)\right) .
\end{aligned}
$$
we get

$$
\begin{aligned}
& { }_{0}^{c} \mathscr{D}_{t}^{\xi} w(T)=\int_{0}^{T} \frac{(T-\eta)^{\varrho^{-\xi}-1}}{\Gamma(\varrho-\xi)} \sigma(\eta) \mathrm{d} \eta-c_{1} \frac{T^{1-\xi}}{\Gamma(2-\xi)}+\left(\frac{T^{1-\xi}}{\Gamma(2-\xi)}\right) \sum_{1=1}^{\mathrm{q}}\left(\frac{\Gamma(2-\nu)}{t_{i}^{1-\nu}} \mathscr{J}_{1}\left(w\left(t_{1}^{-}\right)\right)\right)
\end{aligned}
$$

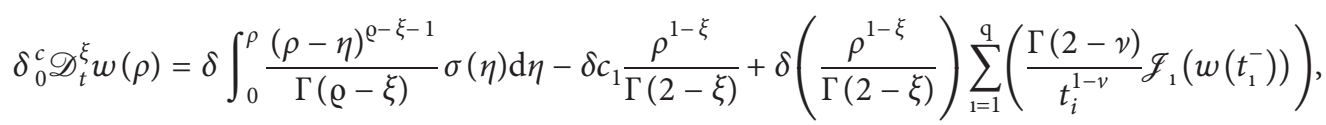

$$
\begin{aligned}
& c_{1} \frac{\left(\delta \rho^{1-\xi}-T^{1-\xi}\right)}{\Gamma(2-\xi)}=\delta \int_{0}^{\rho} \frac{(\rho-\eta)^{\varrho-\xi-1}}{\Gamma(\varrho-\xi)} \sigma(\eta) \mathrm{d} \eta+\delta\left(\frac{\rho^{1-\xi}}{\Gamma(2-\xi)}\right) \sum_{1=1}^{\mathrm{q}}\left(\frac{\Gamma(2-\nu)}{t_{i}^{1-\nu}} \mathcal{J}_{1}\left(w\left(t_{1}^{-}\right)\right)\right) \\
& -\int_{0}^{T} \frac{(T-\eta)^{\varrho-\xi-1}}{\Gamma(\varrho-\xi)} \sigma(\eta) \mathrm{d} \eta-\left(\frac{T^{1-\xi}}{\Gamma(2-\xi)}\right) \sum_{1=1}^{\mathrm{q}}\left(\frac{\Gamma(2-\nu)}{t_{i}^{1-v}} \mathscr{J}_{1}\left(w\left(t_{1}^{-}\right)\right)\right) \\
& c_{1}=\frac{\Gamma(2-\xi)}{\left(\delta \rho^{1-\xi}-T^{1-\xi}\right)}\left[\delta \int_{0}^{\rho} \frac{(\rho-\eta)^{\varrho-\xi-1}}{\Gamma(\varrho-\xi)} \sigma(\eta) \mathrm{d} \eta-\int_{0}^{T} \frac{(T-\eta)^{\varrho-\xi-1}}{\Gamma(\varrho-\xi)} \sigma(\eta) \mathrm{d} \eta\right]+\sum_{1=1}^{\mathrm{q}}\left(\frac{\Gamma(2-\nu)}{t_{i}^{1-\nu}} \mathscr{J}_{1}\left(w\left(t_{1}^{-}\right)\right)\right) .
\end{aligned}
$$

By substituting the value of $c_{1}$ and summarizing, we get the required result.

Conversely, assume that $u$ satisfies the impulsive fractional integral equation (8); then by direct computation, it can be seen that the solution given by (14) satisfies (15).
Corollary 1. In view of the Lemma 2, problem (2) has the following solution:

$$
w(t)=\left\{\begin{array}{l}
\int_{0}^{t} \frac{(t-\eta)^{\varrho-1}}{\Gamma(\varrho)} f\left(\eta, w(\eta),{ }_{0}^{c} \mathscr{D}_{t}^{\varrho} w(\eta)\right) \mathrm{d} \eta-\mathscr{U}, \quad t \in\left[0, t_{1}\right], \\
\int_{0}^{t} \frac{(t-\eta)^{\varrho-1}}{\Gamma(\varrho)} f\left(\eta, w(\eta),{ }_{0}^{c} \mathscr{D}_{t}^{\varrho} w(\eta)\right) \mathrm{d} \eta+\sum_{1=1}^{\mathrm{q}} \mathscr{I}_{1}\left(w\left(t_{1}^{-}\right)\right)-\mathscr{U} \\
+\sum_{1=1}^{\mathrm{q}}\left(t-t_{1}\right)\left(\frac{\Gamma(2-\nu)}{t_{i}^{1-v}} \mathscr{J}_{1}\left(w\left(t_{1}^{-}\right)\right)\right), \quad t \in\left(t_{q}, t_{q+1}\right], q=1,2, \ldots, \mathbf{r},
\end{array}\right.
$$


where

$$
\begin{aligned}
& \mathcal{U}=\frac{\Gamma(2-\xi) t}{\left(\delta \rho^{1-\xi}-T^{1-\xi}\right)} \delta \int_{0}^{\rho} \frac{(\rho-\eta)^{\varrho-\xi-1}}{\Gamma(\varrho-\xi)} f\left(\eta, w(\eta),{ }_{0}^{c} \mathscr{D}{ }_{t}^{\varrho} w(\eta)\right) \mathrm{d} \eta \\
& -\frac{\Gamma(2-\xi) t}{\left(\delta \rho^{1-\xi}-T^{1-\xi}\right)} \int_{0}^{T} \frac{(T-\eta)^{\varrho-\xi-1}}{\Gamma(\varrho-\xi)} f\left(\eta, w(\eta),{ }_{0}^{c} \mathscr{D}_{t}^{\varrho} w\right. \\
& \cdot(\eta)) \mathrm{d} \eta+t \sum_{1=1}^{q}\left(\frac{\Gamma(2-\nu)}{t_{i}^{1-v}} \mathscr{J}_{1}\left(w\left(t_{1}^{-}\right)\right)\right), \quad q=1,2, \ldots, \mathbf{r} .
\end{aligned}
$$

\section{Existence and Uniqueness Results}

In this section, we shall prove our main results. For which, we assume the following assumptions:

$\left(H_{1}\right)$ Let there exist positive constants $L_{1}, L_{2}, L_{3}$, and $L_{4}$ such that for $t \in[0, T]$ and all $w_{1}, w_{2}, h_{1}$, $h_{2} \in \mathscr{R}$, the following inequalities hold:

$$
\begin{aligned}
\mid f\left(t, w_{1}(t),\right. & \left.h_{1}(t)\right)-f\left(t, w_{2}(t), h_{2}(t)\right)\left|\leq L_{1}\right| w_{1}(t)-w_{2}(t)\left|+L_{2}\right| h_{1}(t)-h_{2}(t) \mid, \\
\left|\mathscr{I}_{1}\left(w_{1}\right)\left(t_{1}^{-}\right)-\mathscr{F}_{1}\left(w_{2}\right)\left(t_{1}^{-}\right)\right| & \leq L_{3}\left|w_{1}\left(t_{1}^{-}\right)-w_{2}\left(t_{1}^{-}\right)\right|, \\
& \left|\mathscr{F}_{1}\left(w_{1}\right)\left(t_{1}^{-}\right)-\mathscr{J}_{1}\left(w_{2}\right)\left(t_{1}^{-}\right)\right| \leq L_{4}\left|w_{1}\left(t_{1}^{-}\right)-w_{2}\left(t_{1}^{-}\right)\right| .
\end{aligned}
$$

$\left(H_{2}\right)$ Let the functions $a_{1}, a_{2}, a_{3} \in C\left([0, T], \mathscr{R}^{+}\right)$, which satisfy

$\left|f\left(t, w(t), \mathscr{Y}_{w}(t)\right)\right| \leq a_{1}(t)+a_{2}(t)|w|+a_{3}(t)\left|\mathscr{Y}_{w}(t)\right|$, for $t \in \mathcal{S}, w \in \mathscr{B}$.

such that $a_{3}^{*}=\sup _{t \in[0, T]}\left|a_{3}(t)\right|<1$.

$\left(H_{3}\right)$ If $f, \mathscr{I}_{1}$, and $\mathrm{J}_{1}$ are continuous functions such that for all $w, h \in \mathscr{R}$, the following inequalities hold:

$$
\begin{gathered}
\left|\mathscr{I}_{1}\left(w\left(t_{1}^{-}\right)\right)\right| \leq \mathrm{C}_{\mathcal{F}_{1}}|w|+M_{\mathscr{F}}, \mathrm{C}_{\mathscr{I}_{1}}, M_{\mathscr{I}}>0, \\
\left|J_{1}\left(w\left(t_{1}^{-}\right)\right)\right| \leq \mathrm{C}_{J_{1}}|w|+M_{J}, \mathrm{C}_{J_{1}}, M_{J}>0 .
\end{gathered}
$$

$\left(H_{4}\right)$ Let there exist constants $\beta>0$ and $\epsilon>0$ and a nondecreasing function $\Phi \in C(\mathcal{S}, \mathscr{R})$, such that the following inequalities hold:

$$
{ }_{0} I_{t}^{\mathrm{\rho}} \Phi(t) \leq \beta \Phi(t) \text {; consequently }{ }_{0} I_{t}^{\mathrm{\rho}} \Phi(t) \leq \beta \Phi(t) .
$$

We transform problem (2) into a fixed point problem. Considering an operator $\mathscr{N}: \mathscr{B} \longrightarrow \mathscr{B}$, defined by

$$
(\mathscr{N} w)(t)=\left\{\begin{array}{l}
\int_{0}^{t} \frac{(t-\eta)^{\varrho-1}}{\Gamma(\varrho)} \mathscr{Y}_{w}(\eta) \mathrm{d} \eta-\frac{\Gamma(2-\xi) t}{\left(\delta \rho^{1-\xi}-T^{1-\xi}\right)} \delta \int_{0}^{\rho} \frac{(\rho-\eta)^{\varrho-\xi}-1}{\Gamma(\varrho-\xi)} \mathscr{Y}_{w}(\eta) \mathrm{d} \eta+\frac{\Gamma(2-\xi) t}{\left(\delta \rho^{1-\xi}-T^{1-\xi}\right)} \\
\int_{0}^{T} \frac{(T-\eta)^{\varrho-\xi-1}}{\Gamma(\varrho-\xi)} \mathscr{Y}_{w}(\eta) \mathrm{d} \eta-t \sum_{1=1}^{\mathrm{q}}\left(\frac{\Gamma(2-\nu)}{t_{i}^{1-\nu}} \mathscr{J}_{1}\left(w\left(t_{1}^{-}\right)\right)\right), \quad t \in\left[0, t_{1}\right], q=1,2, \ldots, \mathbf{r}, \\
\int_{0}^{t} \frac{(t-\eta)^{\varrho-1}}{\Gamma(\varrho)} \mathscr{Y}_{w}(\eta) \mathrm{d} \eta-\frac{\Gamma(2-\xi) t}{\left(\delta \rho^{1-\xi}-T^{1-\xi}\right)} \delta \int_{0}^{\rho} \frac{(\rho-\eta)^{\varrho-\xi-1}}{\Gamma(\varrho-\xi)} \mathscr{Y}_{w}(\eta) \mathrm{d} \eta \\
+\frac{\Gamma(2-\xi) t}{\left(\delta \rho^{1-\xi}-T^{1-\xi}\right)} \int_{0}^{T} \frac{(T-\eta)^{\varrho-\xi-1}}{\Gamma(\varrho-\xi)} \mathscr{Y}_{w}(\eta) \mathrm{d} \eta-t \sum_{1=1}^{\mathrm{q}}\left(\frac{\Gamma(2-\nu)}{t_{i}^{1-\nu}} \mathscr{F}_{1}\left(w\left(t_{1}^{-}\right)\right)\right) \\
+\sum_{1=1}^{\mathrm{q}} \mathscr{I}_{1}\left(w\left(t_{1}^{-}\right)\right)+\sum_{1=1}^{\mathrm{q}}\left(t-t_{1}\right)\left(\frac{\Gamma(2-\nu)}{t_{i}^{1-\nu}} \mathscr{F}_{1}\left(w\left(t_{1}^{-}\right)\right)\right), \quad t \in\left(t_{q}, t_{q+1}\right], q=1,2, \ldots, \mathbf{r},
\end{array}\right.
$$


where $\quad \mathscr{Y}_{w}(t)=f\left(t, w(t),{ }_{0}^{c} \mathscr{D}_{t}^{\varrho} w(t)\right)=f(t, w(t)$, $\left.\mathscr{Y}_{w}(t)\right)$. In (40), we see that all the terms of solution $w$ in the interval $\left[0, t_{1}\right]$ are contained in the solution $w$ in interval $\left(t_{q}, t_{q+1}\right]$; therefore, for simplicity purpose, we will study the solution in interval $\left(t_{q}, t_{q+1}\right]$ only. Now, we shall prove some theorems. Our first result is based on Schaefer's fixed theorem.
Theorem 1. If the assumptions $\left(H_{1}\right)-\left(H_{3}\right)$ are satisfied, then problem (2) has at least one solution.

Proof. We use Schaefer's fixed point theorem. The proof is given in the following four steps.

Step 1: to show that $\mathcal{N}$ is continuous, take a sequence $\left\{w_{n}\right\}$ such that $w_{n} \longrightarrow w \in \mathscr{B}$. Then, for $t \in \mathcal{S}$, we have

$$
\begin{aligned}
\left|\mathcal{N}\left(w_{n}(t)\right)-\mathcal{N}(w(t))\right| \leq & \int_{0}^{t} \frac{(t-\eta)^{\varrho-1}}{\Gamma(\varrho)}\left|\mathscr{Y}_{w_{n}}(\eta)-\mathscr{Y}_{w}(\eta)\right| \mathrm{d} \eta \\
& +\frac{t \Gamma(2-\xi)}{\left|\delta \rho^{1-\xi}-T^{1-\xi}\right|} \delta \int_{0}^{\rho} \frac{(\rho-\eta)^{\varrho-\xi-1}}{\Gamma(\varrho-\xi)}\left|\mathscr{Y}_{w_{n}}(\eta)-\mathscr{Y}_{w}(\eta)\right| \mathrm{d} \eta \\
& +\frac{t \Gamma(2-\xi)}{\left|\delta \rho^{1-\xi}-T^{1-\xi}\right|} \int_{0}^{T} \frac{(T-\eta)^{\varrho-\xi-1}}{\Gamma(\varrho-\xi)}\left|\mathscr{Y}_{w_{n}}(\eta)-\mathscr{Y}_{w}(\eta)\right| \mathrm{d} \eta \\
& +t \sum_{1=1}^{q}\left(\frac{\Gamma(2-v)}{t_{i}^{1-v}}\right)\left|\mathscr{J}_{1}\left(w_{n}\left(t_{1}^{-}\right)\right)-\mathscr{J}_{1}\left(w\left(t_{1}^{-}\right)\right)\right|+\sum_{1=1}^{q}\left|\mathscr{I}_{1}\left(w_{n}\left(t_{1}^{-}\right)\right)-\mathscr{I}_{1}\left(w\left(t_{1}^{-}\right)\right)\right| \\
& +\sum_{1=1}^{q}\left|t-t_{1}\right| \frac{\Gamma(2-v)}{t_{i}^{1-v}} \\
& \times\left|\mathscr{J}_{1}\left(w_{n}\left(t_{1}^{-}\right)\right)-\mathscr{J}_{1}\left(w\left(t_{1}^{-}\right)\right)\right|
\end{aligned}
$$

where $\mathscr{Y}_{w_{n}}(t)=f\left(t, w_{n}(t), \mathscr{Y}_{w_{n}}(t)\right)$ and $\mathscr{Y}(t)=f(t$, $\left.w(t), \mathscr{Y}_{w}(t)\right)$. Using $\left(H_{1}\right)$, we have

$$
\begin{aligned}
\left|\mathscr{Y}_{w_{n}}(t)-\mathscr{Y}_{w}(t)\right|= & \mid f\left(t, w_{n}(t), \mathscr{Y}_{w_{n}}(t)\right) \\
& -f\left(t, w(t), \mathscr{Y}_{w}(t)\right) \mid \\
\leq & L_{1}\left|w_{n}(t)-w(t)\right| \\
& +L_{2}\left|\mathscr{Y}_{w_{n}}(t)-\mathscr{Y}_{w}(t)\right|,
\end{aligned}
$$

which implies

$$
\begin{gathered}
\left\|\mathscr{Y}_{w_{n}}-\mathscr{Y}_{w}\right\|_{\mathscr{B}} \leq \frac{L_{1}}{1-L_{2}}\left\|w_{n}-w\right\|_{\mathscr{B}}, \\
\left|\mathscr{J}_{1}\left(w_{n}\right)\left(t_{1}^{-}\right)-\mathscr{J}_{1}(w)\left(t_{1}^{-}\right)\right| \leq L_{4}\left|w_{n}\left(t_{1}^{-}\right)-w\left(t_{1}^{-}\right)\right| .
\end{gathered}
$$

$w_{n} \longrightarrow w$ implies $\mathscr{Y}_{w_{n}}(t) \longrightarrow \mathscr{Y}_{w}(t)$ as $n \longrightarrow \infty$. Now, since every convergent sequence is bounded, there exists a constant $b>0$ such that $\mathscr{Y}_{w_{n}}(t) \leq b$ and $\mathcal{Y}_{w} \leq b$ for $t \in \mathcal{S}$. Thus,

$$
\begin{gathered}
(t-\eta)^{\varrho-1}\left|\mathscr{Y}_{w_{n}}(t)-\mathscr{Y}_{w}(t)\right| \leq(t-\eta)^{\varrho-1}\left(\left|\mathscr{Y}_{w_{n}}(t)\right|+\left|\mathscr{Y}_{w}(t)\right|\right) \leq 2 b(t-\eta)^{\varrho-1}, \\
(T-\eta)^{\varrho-\xi-1}\left|\mathscr{Y}_{w_{n}}(t)-\mathscr{Y}_{w}(t)\right| \leq(T-\eta)^{\varrho-\xi-1}\left(\left|\mathscr{Y}_{w_{n}}(t)\right|+\left|\mathscr{Y}_{w}(t)\right|\right) \leq 2 b(T-\eta)^{\varrho-\xi-1}, \\
(\rho-\eta)^{\varrho-\xi-1}\left|\mathscr{Y}_{w_{n}}(t)-\mathscr{Y}_{w}(t)\right| \leq(\rho-\eta)^{\varrho-\xi-1}\left(\left|\mathscr{Y}_{w_{n}}(t)\right|+\left|\mathscr{Y}_{w}(t)\right|\right) \leq 2 b(\rho-\eta)^{\varrho-\xi-1} .
\end{gathered}
$$

The functions $\eta \longrightarrow 2 b(t-\eta)^{\varrho^{-1}}, \eta \longrightarrow 2 b(T-\eta)^{\mathrm{Q}^{-\xi}-1}$, and $\eta \longrightarrow 2 b(\rho-\eta)^{\varrho^{-\xi-1}}$ are integrable for $t \in \mathcal{S}$. Therefore, by the continuity of $f, \mathscr{I}$, and $\mathscr{J}$ and the Lebesgue dominated convergent theorem, we conclude from $(41)$ that $\left|\mathcal{N}\left(w_{n}(t)\right)-\mathcal{N}(w(t))\right| \longrightarrow 0$ as $n \longrightarrow \infty$ which implies $\left\|\mathscr{N}\left(w_{n}\right)-\mathcal{N}(w)\right\|_{\mathscr{B}} \longrightarrow 0 n \longrightarrow \infty$. This proves the continuity of $\mathcal{N}$.

Step 2: in this step, we will show that for each $w \in \mathscr{W}_{\mathbf{b}}=$ $\{w \in \mathscr{B}:\|w\| \leq \mathbf{b}\},\|\mathcal{N} w\|_{\mathscr{B}} \leq \mathbb{K}$. For $t \in \mathcal{S}$, consider 


$$
\begin{aligned}
|\mathcal{N}(w(t))| \leq & \int_{0}^{t} \frac{(t-\eta)^{\varrho-1}}{\Gamma(\varrho)}\left|\mathscr{Y}_{w}(\eta)\right| \mathrm{d} \eta+\frac{t \Gamma(2-\xi)}{\left|\delta \rho^{1-\xi}-T^{1-\xi}\right|} \delta \int_{0}^{\rho} \frac{(\rho-\eta)^{\varrho-\xi-1}}{\Gamma(\varrho-\xi)}\left|\mathscr{Y}_{w}(\eta)\right| \mathrm{d} \eta \\
& +\frac{t \Gamma(2-\xi)}{\left|\delta \rho^{1-\xi}-T^{1-\xi}\right|} \int_{0}^{T} \frac{(T-\eta)^{\varrho-\xi-1}}{\Gamma(\varrho-\xi)}\left|\mathscr{Y}_{w}(\eta)\right| \mathrm{d} \eta+t \sum_{1=1}^{q}\left(\frac{\Gamma(2-\nu)}{t_{i}^{1-v}}\right)\left|\mathscr{J}_{1}\left(w\left(t_{1}^{-}\right)\right)\right| \\
& +\sum_{1=1}^{q}\left|\mathscr{I}_{1}\left(w\left(t_{1}^{-}\right)\right)\right|+\sum_{1=1}^{q}\left|t-t_{1}\right| \frac{\Gamma(2-\nu)}{t_{i}^{1-v}}\left|\mathscr{J}_{1}\left(w\left(t_{1}^{-}\right)\right)\right| .
\end{aligned}
$$

Using $\left(H_{2}\right)$ and $a_{1}^{*}=\sup _{t \in \mathscr{X}} a_{1}(t), a_{2}^{*}=\sup _{t \in \mathscr{X}} a_{2}(t)$, and $a_{3}^{*}=\sup _{t \in \mathscr{X}} a_{3}(t)<1$, we have

$$
\begin{aligned}
\left|\mathscr{Y}_{w}(t)\right| & =\left|f\left(t, w(t), \mathscr{Y}_{w}(t)\right)\right| \\
& \leq a_{1}(t)+a_{2}(t)|w(t)|+a_{3}(t)\left|\mathscr{Y}_{w}(t)\right| .
\end{aligned}
$$

Taking the maximum value over the interval $\mathcal{S}$ and simplifying, we get

$$
\left\|\mathscr{Y}_{w}\right\|_{\mathscr{B}} \leq \frac{a_{1}^{*}+a_{2}^{*} \mathbf{b}}{1-a_{3}^{*}}=: \mathbb{K}_{1} .
$$

Using this result, (45) implies

$$
\begin{aligned}
|\mathcal{N}(w(t))| \leq & \mathbb{K}_{1} \int_{0}^{t} \frac{(t-\eta)^{\varrho-1}}{\Gamma(\varrho)} \mathrm{d} \eta+\frac{t \Gamma(2-\xi) \mathbb{K}_{1}}{\left|\delta \rho^{1-\xi}-T^{1-\xi}\right|} \delta \int_{0}^{\rho} \frac{(\rho-\eta)^{\varrho-\xi-1}}{\Gamma(\varrho-\xi)} \mathrm{d} \eta \\
& +\frac{t \Gamma(2-\xi) \mathbb{K}_{1}}{\left|\delta \rho^{1-\xi}-T^{1-\xi}\right|} \int_{0}^{T} \frac{(T-\eta)^{\varrho-\xi-1}}{\Gamma(\varrho-\xi)} \mathrm{d} \eta+t \sum_{1=1}^{q}\left(\frac{\Gamma(2-\nu)}{t_{i}^{1-v}}\right)\left(\mathrm{C}_{J_{1}}|w|+M_{J}\right) \\
& +\sum_{1=1}^{q}\left(\mathrm{C}_{\mathcal{I}_{1}}|w|+M_{\mathscr{J}}\right)+\sum_{1=1}^{q}\left|t-t_{1}\right| \frac{\Gamma(2-\nu)}{t_{i}^{1-v}}\left(\mathrm{C}_{J_{1}}|w|+M_{J}\right) .
\end{aligned}
$$

Further simplification implies

$$
\begin{aligned}
\|\mathcal{N} w\|_{\mathscr{B}} \leq & \mathbb{K}_{1}\left(\frac{T^{\varrho}}{\Gamma(\varrho+1)}+\frac{\delta T \Gamma(2-\xi)\left(\delta \rho^{\varrho-\xi}+T^{\varrho^{-\xi}}\right)}{\left|\delta \rho^{1-\xi}-T^{1-\xi}\right| \Gamma(\varrho-\xi+1)}\right) \\
& +2 q T \Gamma(2-\nu)\left(\mathrm{C}_{J_{i}} \mathbf{b}+M_{\mathrm{J}}\right)+\mathrm{q}\left(\mathrm{C}_{\mathcal{F}_{i}} \mathbf{b}+M_{\mathscr{I}}\right)=: \mathbb{K} .
\end{aligned}
$$

This shows that the operator $\mathcal{N}$ maps bounded sets into bounded sets.

Step 3: in this step, we will show that $\mathcal{N}$ is equicontinuous. Let $w \in \mathscr{W} \subseteq \mathscr{B}$ and $t_{1}, t_{2} \in \mathcal{S}$ such that $t_{1}<t_{2}$ and consider

$$
\begin{aligned}
\left|\mathcal{N}\left(w\left(t_{2}\right)\right)-\mathcal{N}\left(w\left(t_{1}\right)\right)\right| \leq & \int_{t_{1}}^{t_{2}} \frac{\left(t_{2}-\eta\right)^{\varrho-1}}{\Gamma(\varrho)}\left|\mathscr{Y}_{w}(\eta)\right| \mathrm{d} \eta+\left(t_{2}-t_{1}\right) \frac{\Gamma(2-\xi)}{\left|\delta \rho^{1-\xi}-T^{1-\xi}\right|} \delta \int_{0}^{\rho} \frac{(\rho-\eta)^{\varrho-\xi-1}}{\Gamma(\varrho-\xi)}\left|\mathscr{Y}_{w}(\eta)\right| \mathrm{d} \eta \\
& +\left(t_{2}-t_{1}\right) \frac{\Gamma(2-\xi)}{\left|\delta \rho^{1-\xi}-T^{1-\xi}\right|} \int_{0}^{T} \frac{(T-\eta)^{\varrho-\xi-1}}{\Gamma(\varrho-\xi)}\left|\mathscr{Y}_{w}(\eta)\right| \mathrm{d} \eta+\left(t_{2}-t_{1}\right) \sum_{0<t_{1}<t_{2}-t_{1}}\left(\frac{\Gamma(2-\nu)}{t_{i}^{1-v}}\right)\left|\mathscr{J}_{1}\left(w\left(t_{1}^{-}\right)\right)\right| \\
& +\sum_{0<t_{1}<t_{2}-t_{1}}\left|\mathscr{I}_{1}\left(w\left(t_{1}^{-}\right)\right)\right|+\sum_{0<t_{1}<t_{2}-t_{1}}\left(t_{2}-t_{1}\right) \frac{\Gamma(2-\nu)}{t_{i}^{1-v}}\left|\mathscr{F}_{1}\left(w\left(t_{1}^{-}\right)\right)\right| .
\end{aligned}
$$


Using assumptions $\left(\mathrm{H}_{2}\right)$ and $\left(\mathrm{H}_{3}\right)$, we obtain

$$
\begin{aligned}
\left|\mathcal{N}\left(w\left(t_{2}\right)\right)-\mathcal{N}\left(w\left(t_{1}\right)\right)\right| \leq & \mathbb{K}_{1} \frac{\left(t_{2}-t_{1}\right)^{\varrho}}{\Gamma(\varrho+1)}+\left(t_{2}-t_{1}\right) \mathbb{K}_{1} \frac{\Gamma(2-\xi)}{\left|\delta \rho^{1-\xi}-T^{1-\xi}\right|} \delta \frac{(\rho)^{\varrho-\xi}}{\Gamma(\varrho-\xi+1)} \\
& +\left(t_{2}-t_{1}\right) \mathbb{K _ { 1 }} \frac{\Gamma(2-\xi)}{\left|\delta \rho^{1-\xi}-T^{1-\xi}\right|} \frac{(T)^{\varrho-\xi}}{\Gamma(\varrho-\xi+1)}+2\left(t_{2}-t_{1}\right) q \Gamma(2-v)\left(\mathrm{C}_{J_{\mathrm{i}}} \mathbf{b}+M_{J}\right) \\
& +\left(t_{2}-t_{1}\right) q \Gamma(2-v)\left(\mathrm{C}_{\mathcal{F}_{i}} \mathbf{b}+M_{\mathscr{I}}\right) .
\end{aligned}
$$

We see that as $t_{1} \longrightarrow t_{2}$, the right-hand side of inequality (51) tends to zero that is $\mid \mathcal{N}\left(w\left(t_{2}\right)\right)-\mathcal{N}(w$ $\left.\left(t_{1}\right)\right) \mid \longrightarrow 0$ as $t_{1} \longrightarrow t_{2}$. Hence, by the Ascoli - Arzela theorem $\mathscr{N}: \mathscr{B} \longrightarrow \mathscr{B}$ is completely continuous.
Step 4: to complete the proof, it remains to show that the set $\mathscr{E}=\{w \in: w=\zeta \mathscr{N} w$, for $0<\zeta<1\}$ is bounded. Let $w \in \mathscr{E}$, then for any $t \in \mathcal{S}$, we have

$$
\begin{aligned}
|\mathcal{N}(w(t))| \leq & \zeta \int_{0}^{t} \frac{(t-\eta)^{\varrho-1}}{\Gamma(\varrho)}\left|\mathscr{Y}_{w}(\eta)\right| \mathrm{d} \eta+\frac{\zeta t \Gamma(2-\xi)}{\left|\delta \rho^{1-\xi}-T^{1-\xi}\right|} \delta \int_{0}^{\rho} \frac{(\rho-\eta)^{\varrho-\xi-1}}{\Gamma(\varrho-\xi)}\left|\mathscr{Y}_{w}(\eta)\right| \mathrm{d} \eta \\
& +\frac{\zeta t \Gamma(2-\xi)}{\left|\delta \rho^{1-\xi}-T^{1-\xi}\right|} \int_{0}^{T} \frac{(T-\eta)^{\varrho-\xi-1}}{\Gamma(\varrho-\xi)}\left|\mathscr{Y}_{w}(\eta)\right| \mathrm{d} \eta+\zeta t \sum_{1=1}^{q}\left(\frac{\Gamma(2-\nu)}{t_{i}^{1-v}}\right)\left|\mathscr{J}_{1}\left(w\left(t_{1}^{-}\right)\right)\right| \\
& +\zeta \sum_{1=1}^{q}\left|\mathscr{I}_{1}\left(w\left(t_{1}^{-}\right)\right)\right|+\zeta \sum_{1=1}^{q}\left|t-t_{1}\right| \frac{\Gamma(2-\nu)}{t_{i}^{1-v}}\left|\mathscr{J}_{1}\left(w\left(t_{1}^{-}\right)\right)\right| .
\end{aligned}
$$

Using $0<\zeta<1$ and (47) and (49), from (52), we get the following result:

$$
\begin{aligned}
\|\mathcal{N} w\|_{\mathscr{B}} \leq & \zeta\left[\mathbb{K}_{1}\left(\frac{T^{\varrho}}{\Gamma(\varrho+1)}+\frac{\delta T \Gamma(2-\xi)\left(\delta \rho^{\varrho^{-\xi}}+T^{\varrho^{-\xi}}\right)}{\left|\delta \rho^{1-\xi}-T^{1-\xi}\right| \Gamma(\varrho-\xi+1)}\right)\right. \\
& \left.+2 q T \Gamma(2-\nu)\left(\mathrm{C}_{J_{i}} \mathbf{b}+M_{\mathrm{J}}\right)+q\left(\mathrm{C}_{\mathscr{F}_{i}} \mathbf{b}+M_{\mathscr{I}}\right)\right]=: \zeta \mathbb{K} \leq \mathbb{K} .
\end{aligned}
$$

This shows that the set $\mathscr{E}$ is bounded. Therefore, by Schaefer's fixed point theorem, problem (2) has at least one solution.

The following and our second result is based on the Banach fixed point theorem.
Theorem 2. If the assumptions $\left(H_{1}\right)-\left(H_{3}\right)$ and the inequality

$$
\begin{aligned}
& \frac{L_{1} T^{\varrho}}{\left(1-L_{2}\right) \Gamma(\varrho+1)}+\frac{L_{1} \delta T \Gamma(2-\xi)\left(\delta \rho^{\varrho-\xi}+T^{\varrho^{-\xi}}\right)}{\left(1-L_{2}\right)\left|\delta \rho^{1-\xi}-T^{1-\xi}\right| \Gamma(\varrho-\xi+1)} \\
& +q\left(L_{3}+2 L_{4} T \Gamma(2-\xi)\right)<1,
\end{aligned}
$$

are satisfied, then (2) has an unique solution.

Proof. To show that the operator $\mathcal{N}$ as defined above has a unique fixed point, we consider $w_{1}, w_{2} \in \mathscr{B}$.

For $\left.t \in t_{\mathrm{q}}, t_{\mathrm{q}+1}\right]$, we have 


$$
\begin{aligned}
\left|\mathcal{N}\left(w_{1}(t)\right)-\mathcal{N}\left(w_{2}(t)\right)\right| \leq & \int_{0}^{t} \frac{(t-\eta)^{\varrho-1}}{\Gamma(\varrho)}\left|\mathscr{Y}_{w_{1}}(\eta)-\mathscr{Y}_{w_{2}}(\eta)\right| \mathrm{d} \eta+\frac{t \Gamma(2-\xi)}{\left(\delta \rho^{1-\xi}-T^{1-\xi}\right)} \\
& \cdot \delta \int_{0}^{\rho} \frac{(\rho-\eta)^{\varrho-\xi-1}}{\Gamma(\varrho-\xi)}\left|\mathscr{Y}_{w_{1}}(\eta)-\mathscr{Y}_{w_{2}}(\eta)\right| \mathrm{d} \eta+\frac{t \Gamma(2-\xi)}{\left(\delta \rho^{1-\xi}-T^{1-\xi}\right)} \int_{0}^{T} \frac{(T-\eta)^{\varrho-\xi-1}}{\Gamma(\varrho-\xi)}\left|\mathscr{Y}_{w_{1}}(\eta)-\mathscr{Y}_{w_{2}}(\eta)\right| \mathrm{d} \eta \\
& +t \sum_{1=1}^{q}\left(\frac{\Gamma(2-\nu)}{t_{1}^{1-\nu}}\right)\left|\mathscr{F}_{1}\left(w_{1}\left(t_{1}^{-}\right)\right)-\mathscr{J}_{1}\left(w_{2}\left(t_{1}^{-}\right)\right)\right|+\sum_{1=1}^{q}\left|\mathscr{I}_{1}\left(w_{1}\left(t_{1}^{-}\right)\right)-\mathscr{I}_{1}\left(w_{2}\left(t_{1}^{-}\right)\right)\right|+\sum_{1=1}^{q}\left|t-t_{1}\right| \frac{\Gamma(2-\nu)}{t_{1}^{1-\gamma}} \\
& \times\left|\mathscr{F}_{1}\left(w_{1}\left(t_{1}^{-}\right)\right)-\mathscr{F}_{1}\left(w_{2}\left(t_{1}^{-}\right)\right)\right|,
\end{aligned}
$$

where $\mathscr{Y}_{w_{1}}(t)=f\left(t, w_{1}(t), \mathscr{Y}_{w_{1}}\right)$ and $\mathscr{Y}_{w_{2}}(\eta)=f\left(t, w_{2}\right.$ $\left.(t), \mathscr{Y}_{w_{2}}\right)$. Using $\left(H_{1}\right)$, we have

$$
\left\|\mathscr{Y}_{w_{1}}-\mathscr{Y}_{w_{2}}\right\|_{\mathscr{B}} \leq \frac{L_{1}}{1-L_{2}}\left\|w_{1}-w_{2}\right\|_{\mathscr{B}}
$$

$$
\begin{aligned}
\left|\mathscr{Y}_{w_{1}}(t)-\mathscr{Y}_{w_{2}}(t)\right| & =\left|f\left(t, w_{1}(t), \mathscr{Y}_{w_{1}}(t)\right)-f\left(t, w_{2}(t), \mathscr{Y}_{w_{2}}(t)\right)\right| \\
& \leq L_{1}\left|w_{1}(t)-w_{2}(t)\right|+L_{2}\left|\mathscr{Y}_{w_{1}}(t)-\mathscr{Y}_{w_{2}}(t)\right|,
\end{aligned}
$$$$
\left|\mathscr{F}_{1}\left(w_{1}\right)\left(t_{1}^{-}\right)-\mathscr{F}_{1}\left(w_{2}\right)\left(t_{1}^{-}\right)\right| \leq L_{4}\left|w_{1}\left(t_{1}^{-}\right)-w_{2}\left(t_{1}^{-}\right)\right| \text {. }
$$

which implies

$$
\begin{aligned}
\left|\mathcal{N}\left(w_{1}\right)(t)-\mathcal{N}\left(w_{2}\right)(t)\right| \leq & \frac{L_{1} T^{\varrho}}{\left(1-L_{2}\right) \Gamma(\varrho+1)} \max _{t \in\left(t_{q}, t_{q+1}\right]}\left|w_{1}(t)-w_{2}(t)\right|+\frac{\Gamma(2-\xi) \rho^{\varrho-\xi}}{\left(1-L_{2}\right)\left|\delta \rho^{1-\xi}-T^{1-\xi}\right| \Gamma(\varrho-\xi+1)} \\
& \cdot \max _{t \in\left(t_{q}, t_{q+1}\right]}(\delta t)\left|w_{1}(t)-w_{2}(t)\right|+\frac{L_{1} \Gamma(2-\xi) T^{\varrho-\xi}}{\left(1-L_{2}\right)\left|\delta \rho^{1-\xi}-T^{1-\xi}\right| \Gamma(\varrho-\xi+1)} \max _{t \in\left(t_{q}, t_{q+1}\right]} t\left|w_{1}(t)-w_{2}(t)\right| \\
& +\max _{t \in\left(t_{q}, t_{q+1}\right]} \frac{q t \Gamma(2-\xi) L_{4}}{t_{q}^{1-\gamma}}\left|w_{1}(t)-w_{2}(t)\right|+\max _{t \in\left(t_{q}, t_{q+1}\right]} q L_{3}\left|w_{1}\left(t_{1}\right)-w_{2}\left(t_{1}\right)\right| \\
& +\max _{t \in\left(t_{q}, t_{q+1}\right]} q\left|t-t_{1}\right| \frac{\Gamma(2-\xi)}{t_{1}^{1-\gamma}} L_{4}\left|w_{1}\left(t_{1}\right)-w_{2}\left(t_{1}\right)\right|, q=1,2, \ldots, \mathbf{r} .
\end{aligned}
$$

By further simplification, we obtain the following inequality:

$$
\begin{aligned}
\left\|\mathcal{N}\left(w_{1}\right)-\mathcal{N}\left(w_{2}\right)\right\|_{\mathscr{B}} \leq & {\left[\frac{L_{1} T^{\varrho}}{\left(1-L_{2}\right) \Gamma(\varrho+1)}+\frac{L_{1} \delta T \Gamma(2-\xi)\left(\delta \rho^{\varrho-\xi}+T^{\varrho^{-} \xi}\right)}{\left(1-L_{2}\right)\left|\delta \rho^{1-\xi}-T^{1-\xi}\right| \Gamma(\varrho-\xi+1)}+q\left(L_{3}+2 L_{4} T \Gamma(2-\xi)\right)\right]\left\|w_{1}-w_{2}\right\|_{\mathscr{B}}, } \\
& \frac{L_{1} T^{\varrho}}{\left(1-L_{2}\right) \Gamma(\varrho+1)}+\frac{L_{1} \delta T \Gamma(2-\xi)\left(\delta \rho^{\varrho-\xi}+T^{Q^{-\xi}}\right)}{\left(1-L_{2}\right)\left|\delta \rho^{1-\xi}-T^{1-\xi}\right| \Gamma(\varrho-\xi+1)}+q\left(L_{3}+2 L_{4} T \Gamma(2-\xi)\right)<1 .
\end{aligned}
$$


Therefore, by Banach contraction theorem, problem (2) has a unique fixed point.

\section{Stability Analysis}

In this section, we study Hyers-Ulam stability of problem (2).

Theorem 3. If assumptions $\left(H_{1}\right)-\left(H_{3}\right)$ and the inequality

$$
\begin{aligned}
& \frac{L_{1} T^{\varrho}}{\left(1-L_{2}\right) \Gamma(\varrho+1)}+\frac{L_{1} \delta T \Gamma(2-\xi)\left(\delta \rho^{\varrho-\xi}+T^{Q^{-\xi}}\right)}{\left(1-L_{2}\right)\left|\delta \rho^{1-\xi}-T^{1-\xi}\right| \Gamma(\varrho-\xi+1)} \\
& \quad+q\left(L_{3}+2 L_{4} T \Gamma(2-\xi)\right)<1,
\end{aligned}
$$

are satisfied, then problem (2) is Hyers-Ulam stable.

Proof. Let $h$ be any solution of inequality (6), then by Remark 1 , we have

$$
\left\{\begin{array}{l}
{ }_{0}^{c} \mathscr{D}_{t}^{\varrho} h(t)=f\left(t, h(t),{ }_{0}^{c} \mathscr{D}_{t}^{e} h(t)\right)+\phi(t), \quad \varrho \in(1,2], \\
\Delta h\left(t_{q}\right)=\mathscr{J}_{q}\left(h\left(t_{q}^{-}\right)\right)+\phi_{q}, \\
\Delta\left({ }_{0}^{c} \mathscr{D}_{t}^{v} h\left(t_{q}\right)\right)=\mathscr{J}_{q}\left(h\left(t_{q}^{-}\right)\right)+\phi_{q}, \quad v \in(0,1], q=1,2, \ldots, \mathbf{r}, \\
h(0)=0,{ }_{0}^{c} \mathscr{D}_{t}^{\xi} h(T)=\delta_{0}^{c} \mathscr{D}_{t}^{\xi} h(\rho)+\phi_{q}, \quad 0<\delta<\xi<1,0<\rho<1, q=1,2, \ldots, \mathbf{r} .
\end{array}\right.
$$

by

In light of Corollary 1, the solution problem (61) is given

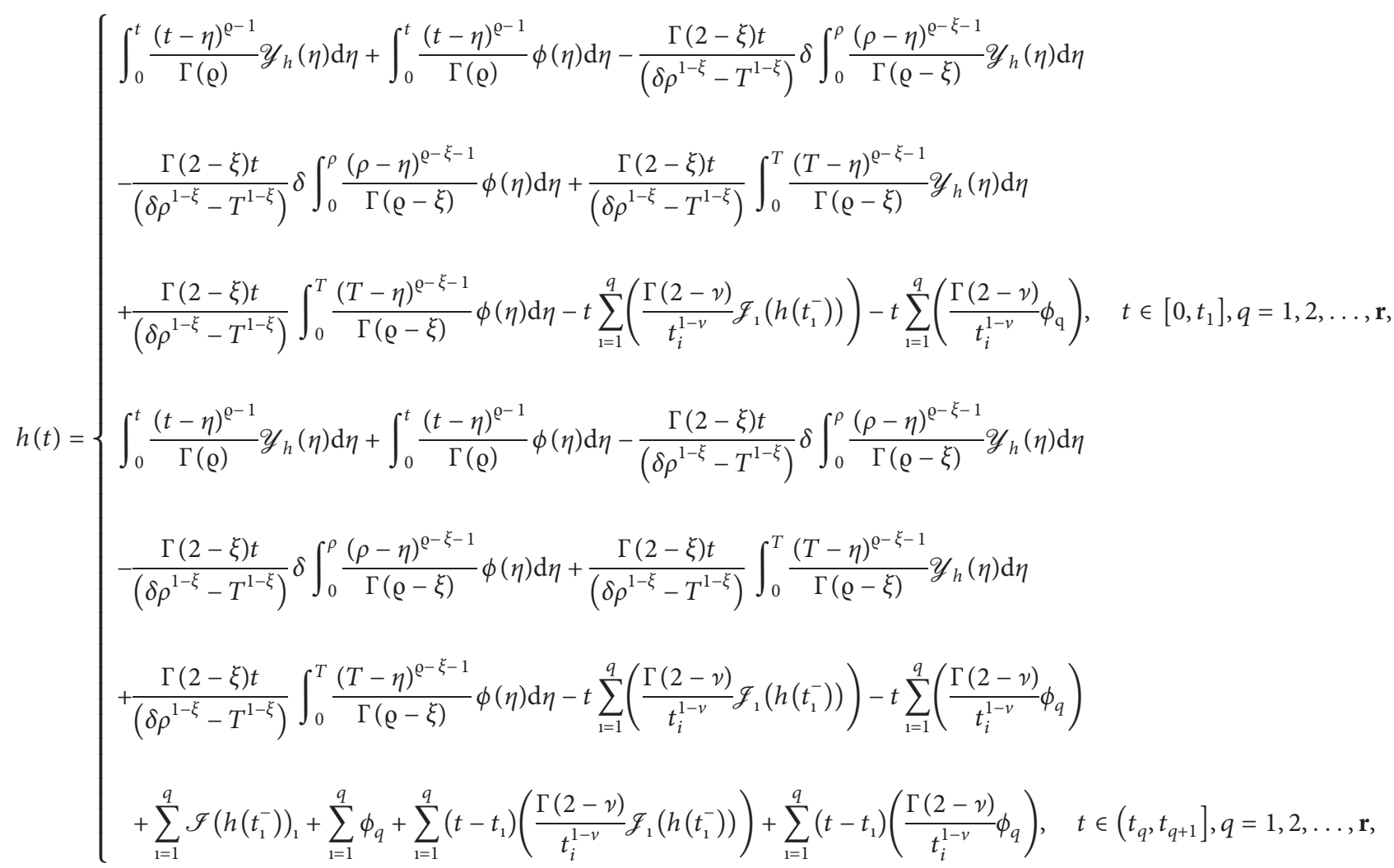


where $\mathscr{Y}_{h}(t)=f\left(t, h(t), \mathscr{Y}_{h}(t)\right)$. Now if $w$ is a unique solution of problem (2), then for $t \in \mathcal{S}$, consider

$$
\begin{aligned}
& |h(t)-w(t)| \leq \int_{0}^{t} \frac{(t-\eta)^{\varrho-1}}{\Gamma(\varrho)}\left|\mathscr{Y}_{h}(\eta)-\mathscr{Y}_{w}(\eta)\right| \mathrm{d} \eta+\int_{0}^{t} \frac{(t-\eta)^{\varrho-1}}{\Gamma(\varrho)}|\phi(\eta)| \mathrm{d} \eta+\frac{\Gamma(2-\xi) t}{\left|\delta \rho^{1-\xi}-T^{1-\xi}\right|} \\
& \cdot \delta \int_{0}^{\rho} \frac{(\rho-\eta)^{\varrho-\xi-1}}{\Gamma(\varrho-\xi)}\left|\mathscr{Y}_{h}(\eta)-\mathscr{Y}_{w}(\eta)\right| \mathrm{d} \eta+\frac{\Gamma(2-\xi) t}{\left|\delta \rho^{1-\xi}-T^{1-\xi}\right|} \delta \int_{0}^{\rho} \frac{(\rho-\eta)^{\varrho-\xi-1}}{\Gamma(\varrho-\xi)}|\phi(\eta)| \mathrm{d} \eta+\frac{\Gamma(2-\xi) t}{\left|\delta \rho^{1-\xi}-T^{1-\xi}\right|} \\
& \cdot \int_{0}^{T} \frac{(T-\eta)^{\varrho-\xi-1}}{\Gamma(\varrho-\xi)}\left|\mathscr{Y}_{h}(\eta)-\mathscr{Y}_{w}(\eta)\right| \mathrm{d} \eta \int_{0}^{T} \frac{(T-\eta)^{\varrho-\xi-1}}{\Gamma(\varrho-\xi)}|\phi(\eta)| \mathrm{d} \eta \\
& +t \sum_{1=1}^{q}\left(\frac{\Gamma(2-v)}{t_{1}^{1-\nu}}\left|\mathscr{J}_{1}\left(h\left(t_{1}^{-}\right)\right)-\mathscr{J}_{1}\left(w\left(t_{1}^{-}\right)\right)\right|\right)+t \sum_{1=1}^{q}\left(\frac{\Gamma(2-\nu)}{t_{1}^{1-\nu}}\left|\phi_{q}\right|\right)+\sum_{1=1}^{q}\left|\mathscr{I}_{1}\left(h\left(t_{1}^{-}\right)\right)-\mathscr{I}_{1}\left(w\left(t_{1}^{-}\right)\right)\right|+\sum_{1=1}^{q}\left|\phi_{q}\right| \\
& +\sum_{1=1}^{q}\left(t-t_{1}\right)\left(\frac{\Gamma(2-v)}{t_{1}^{1-v}}\left|\mathscr{J}_{1}\left(h\left(t_{1}^{-}\right)\right)-\mathscr{J}_{1}\left(w\left(t_{1}^{-}\right)\right)\right|\right)+\sum_{1=1}^{q}\left(t-t_{1}\right)\left(\frac{\Gamma(2-v)}{t_{1}^{1-v}}\left|\phi_{q}\right|\right), \quad t \in\left(t_{q}, t_{q+1}\right], q=1,2, \ldots, \mathbf{r},
\end{aligned}
$$

where $\mathscr{Y}_{w}(t)=f\left(t, w(t), \mathscr{Y}_{w}(t)\right)$. By using assumption $\left(H_{1}\right)-\left(H_{3}\right)$, Remark 1, we have from (63)

$$
\begin{aligned}
\|h-w\|_{\mathscr{B}} \leq & {\left[\frac{L_{1} T^{\varrho}}{\left(1-L_{2}\right) \Gamma(\varrho+1)}+\frac{L_{1} \delta T \Gamma(2-\xi)\left(\delta \rho^{\varrho-\xi}+T^{\varrho^{-} \xi}\right)}{\left(1-L_{2}\right)\left|\delta \rho^{1-\xi}-T^{1-\xi}\right| \Gamma(\varrho-\xi+1)}+q\left(L_{3}+2 L_{4} T \Gamma(2-\xi)\right)\right]\|h-w\|_{\mathscr{B}} } \\
& +\left[\frac{T^{\varrho}}{\Gamma(\varrho+1)}+\frac{\delta T \Gamma(2-\xi)\left(\delta \rho^{\varrho-\xi}+T^{\varrho^{-\xi}}\right)}{\left|\delta \rho^{1-\xi}-T^{1-\xi}\right| \Gamma(\varrho-\xi+1)}+q+2 q T \Gamma(2-\xi)\right] \epsilon .
\end{aligned}
$$

Let

$$
\begin{aligned}
& \mathscr{E}_{1}=\frac{L_{1} T^{\varrho}}{\left(1-L_{2}\right) \Gamma(\varrho+1)}+\frac{L_{1} \delta T \Gamma(2-\xi)\left(\delta \rho^{\varrho-\xi}+T^{\varrho^{-\xi}}\right)}{\left(1-L_{2}\right)\left|\delta \rho^{1-\xi}-T^{1-\xi}\right| \Gamma(\varrho-\xi+1)}+q\left(L_{3}+2 L_{4} T \Gamma(2-\xi)\right), \\
& \mathscr{E}_{2}=\frac{T^{\varrho}}{\Gamma(\varrho+1)}+\frac{\delta T \Gamma(2-\xi)\left(\delta \rho^{\varrho-\xi}+T^{\varrho-\xi}\right)}{\left|\delta \rho^{1-\xi}-T^{1-\xi}\right| \Gamma(\varrho-\xi+1)}+q+2 q T \Gamma(2-\xi),
\end{aligned}
$$

then

$$
\|h-w\|_{\mathscr{B}} \leq \frac{\mathscr{E}_{2}}{1-\mathscr{E}_{1}} \epsilon=\mathscr{C} \epsilon,
$$

where $\mathscr{C}=\mathscr{E}_{2} / 1-\mathscr{E}_{1} ; \mathscr{E}_{1}<1$. This shows that problem (2) is Hyers-Ulam stable. Moreover, by setting $\psi(\epsilon)=\mathscr{C} \epsilon$ and $\psi(0)=0$, then the solution of problem (2) is generalized Hyers-Ulam stable. 
Theorem 4. If assumptions $\left(H_{1}\right)-\left(H_{4}\right)$ and the inequality are satisfied, then problem (2) is Hyers-Ulam-Rassias stable. $\mathscr{E}_{1}=\frac{L_{1} T^{\varrho}}{\left(1-L_{2}\right) \Gamma(\varrho+1)}+\frac{L_{1} \delta T \Gamma(2-\xi)\left(\delta \rho^{\varrho-\xi}+T^{\varrho-\xi}\right)}{\left(1-L_{2}\right)\left|\delta \rho^{1-\xi}-T^{1-\xi}\right| \Gamma(\varrho-\xi+1)} \quad$ Proof. From the proof of Theorem 3, we can write $+q\left(L_{3}+2 L_{4} T \Gamma(2-\xi)\right)<1$

$$
\begin{aligned}
& |h(t)-w(t)| \leq \int_{0}^{t} \frac{(t-\eta)^{\varrho^{-1}}}{\Gamma(\varrho)}\left|\mathscr{Y}_{h}(\eta)-\mathscr{Y}_{w}(\eta)\right| \mathrm{d} \eta+\int_{0}^{t} \frac{(t-\eta)^{\varrho^{-1}}}{\Gamma(\varrho)}|\phi(\eta)| \mathrm{d} \eta \\
& +\frac{\Gamma(2-\xi) t}{\left|\delta \rho^{1-\xi}-T^{1-\xi}\right|} \delta \int_{0}^{\rho} \frac{(\rho-\eta)^{\varrho-\xi-1}}{\Gamma(\varrho-\xi)}\left|\mathscr{Y}_{h}(\eta)-\mathscr{Y}_{w}(\eta)\right| \mathrm{d} \eta+\frac{\Gamma(2-\xi) t}{\left|\delta \rho^{1-\xi}-T^{1-\xi}\right|} \delta \int_{0}^{\rho} \frac{(\rho-\eta)^{\varrho-\xi-1}}{\Gamma(\varrho-\xi)}|\phi(\eta)| \mathrm{d} \eta \\
& +\frac{\Gamma(2-\xi) t}{\left|\delta \rho^{1-\xi}-T^{1-\xi}\right|} \delta \int_{0}^{T} \frac{(T-\eta)^{\varrho-\xi-1}}{\Gamma(\varrho-\xi)}\left|\mathscr{Y}_{h}(\eta)-\mathscr{Y}_{w}(\eta)\right| \mathrm{d} \eta+\frac{\Gamma(2-\xi) t}{\left|\delta \rho^{1-\xi}-T^{1-\xi}\right|} \delta \int_{0}^{T} \frac{(T-\eta)^{\varrho-\xi-1}}{\Gamma(\varrho-\xi)}|\phi(\eta)| \mathrm{d} \eta \\
& +t \sum_{1=1}^{q}\left(\frac{\Gamma(2-v)}{t_{i}^{1-v}}\left|\mathscr{J}_{1}\left(h\left(t_{1}^{-}\right)\right)-\mathscr{J}_{1}\left(w\left(t_{1}^{-}\right)\right)\right|\right)+t \sum_{1=1}^{q}\left(\frac{\Gamma(2-v)}{t_{i}^{1-v}}\left|\phi_{q}\right|\right)+\sum_{1=1}^{q}\left|\mathscr{I}_{1}\left(h\left(t_{1}^{-}\right)\right)-\mathscr{I}_{1}\left(w\left(t_{1}^{-}\right)\right)\right| \\
& +\sum_{1=1}^{q}\left|\phi_{q}\right|+\sum_{1=1}^{q}\left(t-t_{1}\right)\left(\frac{\Gamma(2-v)}{t_{i}^{1-v}}\left|\mathscr{J}_{1}\left(h\left(t_{1}^{-}\right)\right)-\mathscr{J}_{1}\left(w\left(t_{1}^{-}\right)\right)\right|\right)+\sum_{1=1}^{q}\left(t-t_{1}\right)\left(\frac{\Gamma(2-\nu)}{t_{i}^{1-v}}\left|\phi_{q}\right|\right) \\
& t \in\left(t_{q}, t_{q+1}\right], q=1,2, \ldots, \mathbf{r} .
\end{aligned}
$$

By using assumption $\left(H_{1}\right)-\left(H_{4}\right)$, Remark 2, we have from (68)

$$
\begin{aligned}
\|h-w\|_{\mathscr{B}} \leq & \mathscr{E}_{1}\|h-w\|_{\mathscr{B}}+\epsilon\left(\beta+\frac{\delta T \beta \Gamma(2-\xi)}{\left|\delta \rho^{1-\xi}-T^{1-\xi}\right|}+\frac{T \beta \Gamma(2-\xi)}{\left|\delta \rho^{1-\xi}-T^{1-\xi}\right|}\right) \theta(t) \\
& +\epsilon(q+2 q T \Gamma(2-v)) \varphi \leq \mathscr{E}_{1}\|h-w\|_{\mathscr{B}}+\mathscr{E}_{3}(\theta(t)+\varphi) \epsilon
\end{aligned}
$$

which implies that

$$
\begin{gathered}
\|h-w\|_{\mathscr{B}} \leq \frac{\epsilon \mathscr{E}_{3}(\theta(t)+\varphi)}{1-\mathscr{E}_{1}}, \\
\|h-w\|_{\mathscr{B}} \leq \mathscr{C} \epsilon(\theta(t)+\varphi),
\end{gathered}
$$

where

$$
\begin{aligned}
& \mathscr{E}_{1}=\frac{L_{1} T^{\varrho}}{\left(1-L_{2}\right) \Gamma(\varrho+1)}+\frac{L_{1} \delta T \Gamma(2-\xi)\left(\delta \rho^{\varrho^{-\xi}}+T^{Q^{-\xi}}\right)+q\left(L_{3}+2 L_{4} T \Gamma(2-\xi)\right)<1}{\left(1-L_{2}\right)\left|\delta \rho^{1-\xi}-T^{1-\xi}\right| \Gamma(\varrho-\xi+1)}, \\
& \mathscr{E}_{3}=\frac{\beta\left|\delta \rho^{1-\xi}-T^{1-\xi}\right|+\delta T \beta \Gamma(2-\xi)+T \beta \Gamma(2-\xi)}{\left|\delta \rho^{1-\xi}-T^{1-\xi}\right|}+(q+2 q T \Gamma(2-v)) .
\end{aligned}
$$


Therefore, problem (2) is Hyers-Ulam-Rassias stable.

Example 1

\section{Example}

Consider the following implicit fractional system with given impulsive conditions.

$$
\left\{\begin{array}{l}
{ }_{0}^{c} \mathscr{D}_{t}^{3 / 2} w(t)=\frac{|w(t)|}{26(t+1 / 2)(1+|w(t)|)}+\frac{\sin \left|{ }_{0}^{c} \mathscr{D}_{t}^{3 / 2} w(t)\right|}{26+t^{3}}, \quad t \in \mathcal{S}=[0,1], t \neq t_{1}, q=\mathbf{r}=1, \\
w(0)=0 \\
{ }_{0}^{c} \mathscr{D}_{t}^{1 / 2} w(1)=\frac{1}{4}\left(\frac{12+|w(\rho)|+\left|{ }_{0}^{c} \mathscr{D}_{t}^{1 / 2} w(\rho)\right|}{72 e^{\rho+3}\left(1+|w(\rho)|+\left|{ }_{0}^{c} \mathscr{D}_{t}^{1 / 2} w(\rho)\right|\right)}\right)_{\rho=1 / 5}, \\
\Delta w\left(t_{1}\right)=\frac{\left|w\left(t_{1}^{-}\right)\right|}{40+\left|w\left(t_{1}^{-}\right)\right|} \\
\Delta_{0}^{c} \mathscr{D}_{t}^{1 / 2} w\left(t_{1}\right)=\frac{\left|w\left(t_{1}^{-}\right)\right|}{32+\left|w\left(t_{1}^{-}\right)\right|} .
\end{array}\right.
$$

Obviously, the given function $f$ is continuous. We see $\varrho=5 / 4, \quad \xi=\nu=1 / 2, \quad T=1, \quad \delta=1 / 4$, and $\rho=1 / 5$. For $w_{1}, w_{2} \in \mathscr{B}$ and $\mathscr{Y}_{w_{1}}, \mathscr{Y}_{w_{2}} \in C(\mathcal{S}, \mathscr{R})$ and $t \in \mathcal{S}$,

$$
\begin{aligned}
& \left|f\left(t, w_{1}(t), \mathscr{Y}_{w_{1}}\right)-f\left(t, w_{2}(t), \mathscr{Y}_{w_{2}}\right)\right| \\
& \quad \leq \frac{1}{26}\left(\left|w_{1}(t)-w_{2}(t)\right|+\left|\mathscr{Y}_{w_{1}}-\mathscr{Y}_{w_{2}}\right|\right),
\end{aligned}
$$

which satisfies $\left(H_{1}\right)$ with $L_{1}=L_{2}=1 / 26$. Further, for $t_{1}=1 / 5$, let

$$
\begin{aligned}
& \mathscr{I}_{1}\left(w\left(t_{1}^{-}\right)\right)=\frac{\left|w\left(t_{1}^{-}\right)\right|}{40+\left|w\left(t_{1}^{-}\right)\right|}, \\
& \mathscr{J}_{1}\left(w\left(t_{1}^{-}\right)\right)=\frac{\left|w\left(t_{1}^{-}\right)\right|}{32+\left|w\left(t_{1}^{-}\right)\right|}, \quad \text { where } w_{1} \in \mathscr{B} .
\end{aligned}
$$

For any $w_{1}, w_{2} \in \mathscr{B}$, we have

$$
\begin{aligned}
\left|\mathscr{I}\left(w_{1}\left(t_{1}^{-}\right)\right)-\mathscr{I}\left(w_{2}\left(t_{1}^{-}\right)\right)\right| & =\left|\frac{\left|w\left(t_{1}^{-}\right)\right|}{40+\left|w\left(t_{1}^{-}\right)\right|}-\frac{\left|w\left(t_{1}^{-}\right)\right|}{40+\left|w\left(t_{1}^{-}\right)\right|}\right| \\
& \leq \frac{1}{40}\left|w_{1}\left(t_{1}^{-}\right)-w_{2}\left(t_{1}^{-}\right)\right|, \\
\left|\mathscr{I}\left(w_{1}\left(t_{1}^{-}\right)\right)-\mathscr{I}\left(w_{2}\left(t_{1}^{-}\right)\right)\right| & =\left|\frac{\left|w\left(t_{1}^{-}\right)\right|}{32+\left|w\left(t_{1}^{-}\right)\right|}-\frac{\left|w\left(t_{1}^{-}\right)\right|}{32+\left|w\left(t_{1}^{-}\right)\right|}\right| \\
& \leq \frac{1}{32}\left|w_{1}\left(t_{1}^{-}\right)-w_{2}\left(t_{1}^{-}\right)\right|,
\end{aligned}
$$

which satisfy the 2 nd and 3 rd inequalities of $\left(H_{1}\right)$ with $L_{3}=1 / 40$ and $L_{4}=1 / 32$.

For $\varrho=1 / 5, \quad \xi=v=1 / 2, T=1, \quad \delta=1 / 4, \rho=1 / 4, L_{1}=$ $L_{2}=1 / 26, L_{3}=1 / 40$, and $L_{4}=1 / 32$, we have

$$
\begin{aligned}
\mathscr{E}_{1}= & \frac{L_{1} T^{\varrho}}{\left(1-L_{2}\right) \Gamma(\varrho+1)}+\frac{\left.L_{1} \delta T \Gamma(2-\xi)\left(\delta \rho^{\varrho-\xi}+T^{\varrho^{-\xi}}\right)\right)}{\left(1-L_{2}\right)\left|\delta \rho^{1-\xi}-T^{1-\xi}\right| \Gamma(\varrho-\xi+1)} \\
& +q\left(L_{3}+2 L_{4} T \Gamma(2-\xi)\right)=0.301+0.009+0.08=0.39<1 .
\end{aligned}
$$

Therefore, by Theorem 2, problem (72) has a unique solution. By Theorem 4, problem (52) is Hyers-Ulam stable and consequently generalized Hyers-Ulam stable.

Further, assuming $\Phi(t)=1$, we have

$$
{ }_{0} I_{1}^{3 / 2} \Phi(t)=\frac{1}{\Gamma(3 / 2)} \int_{0}^{1}(1-\eta)^{(3 / 2)-1} \eta \mathrm{d} \eta \leq \frac{1}{3 \sqrt{\Pi}}
$$

Consequently,

$$
{ }_{0} I_{1}^{(3 / 2)-1} \Phi(t)=\frac{1}{\Gamma(3 / 2-1)} \int_{0}^{1}(1-\eta)^{(3 / 2)-2} \eta \mathrm{d} \eta \leq \frac{1}{3 \sqrt{\Pi}},
$$

which satisfies $\left(H_{4}\right)$ with $\beta=1 / 3 \sqrt{\Pi}$ and $\Phi(t)=1$; therefore, by Theorem 4, the solution of (72) is Hyers-UlamRassias stable corresponding to $(\Phi, \psi)$. With the help of Matlab, we plot the result by using the RK4 method in Figure 1. 


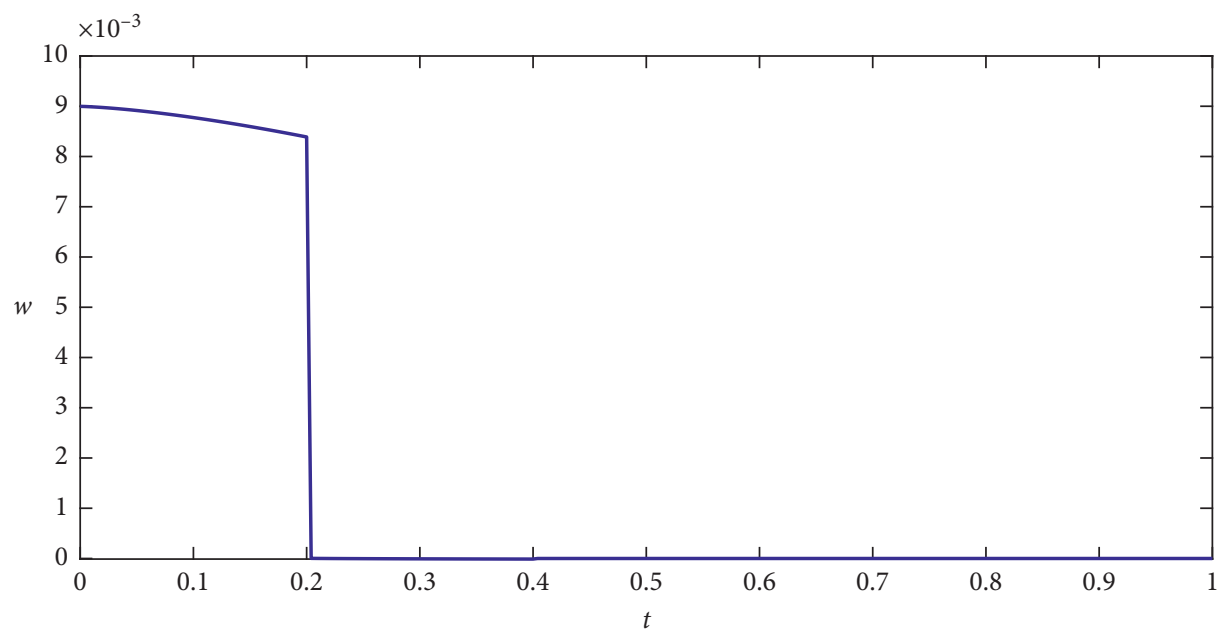

FIgURE 1: Graphical presentation of result of Example 1.

\section{Conclusion}

In this paper, using Schaefer's fixed point theorem, we have derived sufficient conditions for at least one solution to a class of IFODEs under NCFBC 2. Similarly, using the Banach contraction theorem, we obtained conditions under which problem 2 has unique solution. Moreover, by the application of qualitative theory and nonlinear functional analysis, we established results concerning to various kinds of Hyers-Ulam stability. The concerned results have been verified by a suitable example.

\section{Data Availability}

No data were used to support this study.

\section{Conflicts of Interest}

The authors declare that they have no conflicts of interest regarding publication of this paper.

\section{Authors' Contributions}

All authors have equal contribution in this research paper.

\section{Acknowledgments}

The third author T. Abdeljawad would like to thank Prince Sultan University for funding this work through the research group Nonlinear Analysis Methods in Applied Mathematics (NAMAM), group number RG-DES-2017-01-17.

\section{References}

[1] A. A. Kilbas, H. M. Srivastava, and J. J. Trujillo, Theory and Applications of Fractional Diffrential Equations, Vol. 204, North-Holland Mathematics Studies, Elsevier, Amsterdam, Netherlands, 2006.

[2] A. A. Kilbas, O. I. Marichev, and S. G. Samko, Fractional Integral and Derivatives (Theory and Applications), Gordon \& Breach, Basel, Switzerland, 1993.
[3] R. Hilfer, "Fractional time evolution," Applications of Fractional Calculus in Physics, World Scientific, Singapore, vol. 35, no. 12, pp. 87-130, 2000.

[4] P. J. Torvik and R. L. Bagley, "On the appearance of the fractional derivative in the behavior of real materials," Journal of Applied Mechanics, vol. 51, no. 2, pp. 294-298, 1984.

[5] R. A. Khan and K. Shah, "Existence and uniqueness of solutions to fractional order multi-point boundary value problems," Communications in Applied Analysis, vol. 19, pp. 515-526, 2015.

[6] A. Ali and K. Shah, "Existence theory and Ulam-Hyers stability to anti-periodic integral boundary value problem of implicit fractional differential equations," Applied Mathematics E-Notes, vol. 19, pp. 228-242, 2019.

[7] J. V. D. C. Sousa and E. C. de Oliveira, "On the $\psi$-Hilfer fractional derivative," Communications in Nonlinear Science and Numerical Simulation, vol. 60, pp. 72-91, 2018.

[8] J. V. D. C. Sousa and E. C. de Oliveira, "Leibniz type rule: $\psi$-Hilfer fractional operator," Communications in Nonlinear Science and Numerical Simulation, vol. 77, pp. 305-311, 2019.

[9] A. Khan, M. I. Syam, A. Zada, and H. Khan, "Stability analysis of nonlinear fractional differential equations with Caputo and Riemann-Liouville derivatives," The European Physical Journal Plus, vol. 133, no. 7, pp. 1-9, 2018.

[10] J. V. D. C. Sousa and E. C. de Oliveira, "Ulam-Hyers stability of a nonlinear fractional Volterra integro-differential equation," Applied Mathematics Letters, vol. 81, pp. 50-56, 2018.

[11] K. Shah, A. Ali, and S. Bushnaq, "Hyers-Ulam stability analysis to implicit Cauchy problem of fractional differential equations with impulsive conditions," Mathematical Methods in the Applied Sciences, vol. 41, no. 17, pp. 1-15, 2018.

[12] N. Ahmad, Z. Ali, K. Shah, A. Zada, and G. ur Rahman, "Analysis of implicit type nonlinear dynamical problem of impulsive fractional differential equations," Complexity, vol. 2018, pp. 1-15, 2018.

[13] A. Ali, K. Shah, and D. Baleanu, "Ulam stability results to a class of nonlinear implicit boundary value problems of impulsive fractional differential equations," Advances in Difference Equations, vol. 2019, no. 5, pp. 1-21, 2019.

[14] A. Zada and S. Ali, "Stability of integral Caputo-type boundary value problem with noninstantaneous impulses," International Journal of Applied and Computational Mathematics, vol. 5, no. 3, pp. 1-55, 2019. 
[15] A. Ali, K. Shah, F. Jarad, V. Gupta, and T. Abdeljawad, "Existence and stability analysis to a coupled system of implicit type impulsive boundary value problems of fractionalorder differential equations," Advances in Difference Equations, vol. 2019, no. 101, pp. 1-21, 2019.

[16] A. Zada and S. Ali, "Stability analysis of multi-point boundary value problem for sequential fractional differential equations with non-instantaneous impulses," International Journal of Nonlinear Sciences and Numerical Simulation, vol. 19, no. 7-8, pp. 763-774, 2018.

[17] V. Gupta and J. Dabas, "Existence results for a fractional integro-differential equation with nonlocal boundary conditions and fractional impulsive conditions," Nonlinear Dynamics and Systems Theory, vol. 15, no. 4, pp. 370-382, 2015.

[18] R. P. Agarwal, M. Benchohra, and S. Hamani, "A survey on existence results for boundary value problems of nonlinear fractional differential equations and inclusions," Acta Applicandae Mathematicae, vol. 109, no. 3, pp. 973-1033, 2010.

[19] I. A. Rus, "Ulam stabilities of ordinary differential equations in a Banach space," Carpathian Journal of Mathematics, vol. 26, pp. 103-107, 2010.

[20] A. Cabada and G. Wang, "Positive solutions of nonlinear fractional differential equations with integral boundary value conditions," Journal of Mathematical Analysis and Applications, vol. 389, no. 1, pp. 403-411, 2013. 